\title{
Computer simulation study of gas-liquid nucleation in a Lennard-Jones system
}

\author{
Pieter Rein ten Wolde and Daan Frenkel \\ FOM Institute for Atomic and Molecular Physics, Kruislaan 407, 1098 SJ Amsterdam, The Netherlands
}

(Received 12 June 1998; accepted 1 September 1998)

\begin{abstract}
We report a computer-simulation study of homogeneous gas-liquid nucleation in a Lennard-Jones system. Using umbrella sampling, we compute the free energy of a cluster as a function of its size. A thermodynamic integration scheme is employed to determine the height of the nucleation barrier as a function of supersaturation. Our simulations illustrate that the mechanical and the thermodynamical surfaces of tension and surface tension differ significantly. In particular, we show that the mechanical definition of the surface tension cannot be used to compute this barrier height. We find that the relations recently proposed by McGraw and Laaksonen [J. Chem. Phys. 106, 5284 (1997)] for the height of the barrier and for the size of the critical nucleus are obeyed. (C) 1998 American Institute of Physics. [S0021-9606(98)50946-4]
\end{abstract}

\section{INTRODUCTION}

The spontaneous formation of liquid droplets in a supersaturated vapor is probably the best known example of homogeneous nucleation. In view of its great practical importance, a large number of experimental studies of gas-liquid nucleation has been reported and the earliest theoretical description dates back to the 1920 s. $^{1,2}$

In recent years, research on gas-liquid nucleation has received a new impetus. This is partly due to the emergence of sophisticated experimental techniques that make it possible to measure nucleation rates with unprecedented accuracy. ${ }^{3,4}$ The so-called nucleation theorem ${ }^{3,5-7}$ makes it possible to deduce, from the experimental data on the nucleation rate, detailed information about the size and composition of the critical nucleus. ${ }^{3,8-10}$ In parallel, modifications and extensions of the classical nucleation theory have been proposed $^{11-15}$ and novel theoretical tools ${ }^{16}$ have made it possible to go beyond the essentially macroscopic description that lies at the basis of classical nucleation theory. In particular, expressions for the height of the barrier and the size of the critical nucleus have been proposed that could provide a possible explanation for the systematic discrepancies between classical nucleation theory (CNT) and experiment. ${ }^{17,18}$ Furthermore, much theoretical progress has been made in calculating the Tolman length, ${ }^{19}$ which is an important quantity in nucleation as it describes how the surface free energy changes with droplet size.

However, despite the fact that nucleation rate measurements have become increasingly accurate, many of the theoretical predictions are difficult to test directly in an experiment as they are concerned with the microscopic structure of the critical nucleus. Computer simulation is a natural tool to study the details of the nucleation process, because it yields essentially exact microscopic information about the model studied.

Liquid clusters in vapor have been studied in detail by computer simulation. ${ }^{20-27}$ Most of these simulations were done in the canonical ensemble, that is, at constant number of particles $N$, constant temperature $T$, and constant volume
$V$. A simple analysis ${ }^{22,25}$ of the change in Helmholtz free energy when a liquid droplet is formed from the vapor shows that a liquid cluster can be in equilibrium with the vapor in a constant volume simulation. However, in most nucleation experiments the pressure or, equivalently, the chemical potential of the vapor, is held constant. Classical nucleation theory also usually considers the nucleation of a liquid drop from the vapor at constant chemical potential, i.e., at constant pressure of the vapor. Therefore, one would like to perform a simulation at constant pressure rather than at constant volume. At constant pressure a liquid cluster can be in equilibrium with the vapor at the top of the nucleation barrier. However, this equilibrium is unstable. If a cluster, due to spontaneous fluctuations, becomes larger than the critical size, it will grow, because in that way it can minimize its excess free energy. On the other hand, if a cluster by chance becomes smaller than this critical size, it will shrink, again because in that way it can lower its excess free energy.

Hence, in a standard NPT simulation it is virtually impossible to study a critical cluster. However, using the umbrella-sampling scheme $\mathrm{s}^{28}$ it is possible to stabilize the critical cluster at constant pressure. But, more importantly, it also makes it possible to stabilize the precritical (and postcritical) nuclei, thus allowing us to compute the excess free energy of a cluster as a function of its size at constant pressure. Hence, with the umbrella-sampling technique we obtain not only structural information about the precritical and critical nuclei, but also about the height of the nucleation barrier. Moreover, the umbrella sampling technique can be combined with a thermodynamic integration scheme, which enables the efficient computation of the height of the barrier as a function of supercooling.

This integration scheme is not only efficient, but also very accurate and allows us to test several theoretical predictions. First, we examine to what extent the nuclei behave like small droplets of bulk liquid, which is one of the main assumptions of classical nucleation theory. We then compare the size of the critical nuclei and the height of the nucleation barrier with the corresponding predictions of classical nucle- 
ation theory. Furthermore, we have investigated whether the deviations from classical nucleation theory can be accounted for by a curvature correction to the surface tension, as proposed by McGraw and Laaksonen. ${ }^{17,18}$

We have also computed the Tolman length, ${ }^{29}$ which describes the lowest-order correction to the surface tension. As will be discussed in more detail below, the Tolman length is defined as the difference in position between the equimolar dividing surface and the thermodynamically defined surface of tension. It is possible to give both a mechanical definition and a thermodynamic definition of the surface tension and surface of tension of a liquid droplet (see Refs. 30, 31). However, we find that both definitions are not equivalent, which means that we cannot obtain the Tolman length from the mechanical description of the droplet. More importantly, this also implies that we cannot use a simple "mechanical" expression to compute the height of the nucleation barrier. Our simulations illustrate the fact that the thermodynamic and mechanical definitions cannot be used interchangeably, and that any attempt to do so leads to an incorrect estimate of the height of the nucleation barrier.

We have performed our simulations for a Lennard-Jones system. The choice for this system was motivated by several factors. First, the phase behavior of this system is known. ${ }^{32}$ Second, gas-liquid nucleation in this system has also been studied extensively with density functional theory. ${ }^{33-35} \mathrm{Fi}-$ nally, the Tolman length has been computed both numerically and theoretically for the Lennard-Jones system. ${ }^{19,26,36}$

The rest of the paper is organized as follows. In the next section, we give relations for the cluster-size distributions in terms of the free energy of the clusters. We then describe the numerical techniques to calculate the cluster-size distributions and the nucleation barriers. In Sec. IV we discuss how we can obtain the mechanically and thermodynamically defined surface tension and surface of tension. We then give the computational details of the simulations in Sec. V, and in Sec. VI we discuss the results.

\section{CLUSTER-SIZE DISTRIBUTION}

In order to perform a numerical study of the formation of a liquid-like droplet from the vapor phase, we need an unambiguous definition of an incipient liquid-like cluster. In the following, we use an approach that is quite similar, but not quite identical, to the one introduced more than 30 years ago by Stillinger. ${ }^{37}$ Consider a vapor in a constant volume $V$, at constant temperature $T$, and at constant chemical potential $\mu$. In gas-liquid nucleation, the density of liquid-like clusters is usually so low that the interactions between them can be neglected. Furthermore, let us assume that we have a criterion that enables us to define which particles make up a liquid cluster. As shown in Appendix A, the average number $N_{n}$ of clusters of size $n$ is then given by

$$
\left\langle N_{n}\right\rangle=Z_{n} \exp [\beta \mu n] .
$$

Here, $\beta \equiv 1 / k_{\mathrm{B}} T$ is the reciprocal temperature, $k_{\mathrm{B}}$ is Boltzmann's constant, and $Z_{n}$ is the partition function of the $n$ mer. It is given by

$$
Z_{n}=\frac{V n^{3}}{\Lambda^{3 n} n !} \int d r^{\prime n-1} \exp \left[-\beta W_{n}\left(r^{\prime n-1} ; \mu\right)\right] .
$$

Here, $\Lambda \equiv h / \sqrt{2 \pi m k_{\mathrm{B}} T}$ is the thermal De Broglie wavelength, $r^{\prime n-1}$ denotes the coordinates with the prime indicating that the coordinates are taken with respect to the centerof-mass of the cluster, and $W$ is the potential of mean force. The potential of mean force is obtained by carrying out the integration over the coordinates of the vapor particles. That is, all possible configurations of the vapor particles will contribute to $W$; it is the average potential the particles in the liquid clusters feel, due to all interactions with the "solvent" particles. As shown in Appendix A, our cluster criterion is absorbed into the definition of the potential of mean force. All configurations of the particles in the cluster that do not satisfy the cluster criterion will not contribute to the partition function. To illustrate this, let us consider a cluster of noninteracting particles. The partition function of such an $n$-mer is

$$
Z_{n}=\frac{V n^{3}}{\Lambda^{3 n} n !} \int d r^{\prime n-1} w\left(r^{n-1}\right),
$$

where the function $w\left(r^{n-1}\right)$ is defined to be one, if its arguments satisfy the cluster criterion, and zero otherwise. Note that the volume of this "ideal-gas cluster" is well defined and that the cluster criterion provides a natural volume scale for the cluster. ${ }^{38}$

If we define the free-energy of an $n$-mer as

$$
F_{n} \equiv-k_{\mathrm{B}} T \ln Z_{n},
$$

Eq. (1) can be rewritten as

$$
\left\langle N_{n}\right\rangle=\exp \left[-\beta\left(F_{n}-n \mu\right)\right]=\exp [-\beta \Delta F] .
$$

An expression very similar to the one above has been obtained by Reiss et al. ${ }^{27,39-43}$ The only difference is that we make fewer assumptions; Reiss et al. assumed that the surrounding gas is ideal and that there is no interaction between molecules that are inside the cluster and those outside. Instead, we have carried out the integration over the coordinates of the noncluster (solvent) particles, and the effect of the surrounding phase is adsorbed into the effective interactions between the particles that make up the cluster. Note also that, in the present description, we need not introduce the concept of a "shell-molecule" to arrive at an unique definition of the clusters ${ }^{27,40-43}$ - whether or not a molecule belongs to a cluster is uniquely defined by our cluster criterion. In particular, the present approach allows for monomeric clusters. Of course, we still have to specify the cluster criterion. Our choice for this function is described in detail in Sec. V. Here, it suffices to say that it enables us to identify which particles in the system have a liquid-like density; all liquid-like particles that are connected make up a cluster. An added advantage is that the present approach does not suffer from the problem of redundant counting of configuration space. ${ }^{38-40}$ Furthermore, the present scheme can easily be used to study crystal nucleation. ${ }^{44,45}$ In crystal nucleation, the density difference between the crystallite and the surrounding liquid is usually so low that the interactions between the cluster and the surrounding medium cannot be ignored. 
In practice, it is useful to express the number of clusters of size $n$ (which is extensive) in terms of a probability (which is intensive)

$$
P_{n} \equiv \frac{N_{n}}{N},
$$

where $N$ is the total number of particles in the system. This, in turn, defines an intensive Gibbs free-energy of the cluster (where the reference state is the homogeneous phase):

$$
\Delta G_{n} \equiv-k_{\mathrm{B}} T \ln \left[P_{n}\right] .
$$

The average number of clusters of size $n$ is then given by

$$
\left\langle N_{n}\right\rangle=N \exp \left(-\beta \Delta G_{n}\right) .
$$

\section{NUCLEATION BARRIER}

\section{A. The free-energy barrier as a function of droplet size}

The probability distribution function $P(n)$, as defined in Eq. (6), is an equilibrium property and can be measured both by Monte Carlo (MC) and molecular dynamics (MD). However, at experimentally accessible degrees of supersaturation, the brute force approach, in which we would simulate the supersaturated vapor, either by MC or MD, and simply count the liquid clusters that spontaneously appear, would never yield an accurate measure of the nucleation barrier. In experiments, the height of the nucleation barrier is typically in the order of $75 k_{\mathrm{B}} T$. This means that the probability of finding a cluster that has the critical size is extremely small, of the order of $10^{-30}$. Hence, the numerical accuracy of any direct simulation will be very poor. To obtain good statistics for all values of $n$, we therefore use the umbrella sampling scheme of Torrie and Valleau. ${ }^{28}$ The basic idea of this scheme is to bias the sampling of configuration space and correct for the bias afterward.

We can bias the sampling by adding a fictitious potential to the true potential of our original system. In a previous study ${ }^{46,47}$ on crystal nucleation in a Lennard-Jones system, we used a biasing potential that was a function of a global order parameter, $Q_{6}$. This order parameter measured the overall degree of crystallinity in the system and served as a reaction coordinate from the liquid to the solid. By using the biasing potential, we could move the system along the reaction coordinate from the liquid to the solid, and vice versa. In the same spirit, we could now apply a biasing potential that depends on an order parameter which is sensitive to the total number of liquid particles in the supersaturated vapor. By increasing the value of this order parameter, using the biasing potential, we could then cross the nucleation barrier and force the system to condense.

However, as explained in detail in Ref. 44, the use of a global order parameter has some serious drawbacks from a computational point of view. The reason is the following: if we use the total amount of the new phase as a reaction coordinate, then the value of this reaction coordinate tells us how much of the new phase (say, liquid) we have, but not how it is distributed in space. In particular, the new phase need not be concentrated in one cluster. In fact, in a sufficiently large volume it is always entropically favorable for a cluster to break up in smaller fragments. However, for the nucleation process, we are interested in the properties of the largest connected cluster.

Rather than using a global order parameter, we therefore use a local order parameter. We define the order parameter to be the size, $n$, of the largest liquid cluster present in the system. The advantage of this scheme is that by using a biasing potential which is a function of this order parameter, we can directly control the size of one cluster and sample all sizes of this cluster with equal accuracy. The functional form of the biasing potential $W$ was taken to be harmonic,

$$
W\left[n\left(\mathbf{r}^{N}\right)\right]=\frac{1}{2} k_{n}\left[n\left(\mathbf{r}^{N}\right)-n_{0}\right]^{2},
$$

where $\mathbf{r}^{N}$ denotes the atomic coordinates. The result of adding this potential to the true potential of our model system is that, in every run, a "window" of cluster sizes will be sampled. The width and "location" of this window depend on $k_{n}$ and $n_{0}$. By increasing $n_{0}$ we can increase the size of the cluster.

\section{B. The free-energy barrier as a function of supersaturation}

In principle, one could compute the height of the freeenergy barrier for every degree of supersaturation by the scheme discussed in the previous section. However, calculating the free-energy curve all the way up to the top of the barrier is rather time consuming because a lot of windows have to be simulated.

We therefore followed a different approach. Only for one pressure did we compute the full nucleation barrier by the umbrella-sampling technique as outlined in the previous section. This free-energy barrier is then used as a reference for the calculation of the height of the free-energy barrier at other supersaturations. The main idea is that for every pressure we only have to perform two simulations: one in the metastable vapor phase and one at the top of the barrier. For both states we can determine the variation of the free energy with pressure, from which we obtain how the height of the nucleation barrier changes with pressure. By linking the variation of the barrier height with the height of the barrier at the reference pressure, we then obtain the height of the nucleation barrier as a function of total pressure.

To make this more explicit, consider the (Landau) Gibbs free-energy $G$, which is a function of the number of particles $N$, the pressure $P$, the temperature $T$, and the size of the cluster $n$. The first differential of the Gibbs free-energy is

$d G(N, P, T, n)$

$$
=\mu(n) d N+V(n) d P-S(n) d T+\left.\frac{\partial G}{\partial n}\right|_{N, P, T} d n,
$$

where $\mu$ is the chemical potential, $V$ is the volume, and $S$ is the entropy of the system. At a constant total number of particles and temperature, Eq. (9) reduces to

$$
d G(N, P, T, n)=V(n) d P+\left.\frac{\partial G}{\partial n}\right|_{N, P, T} d n .
$$


Using the above equation, we can now compute the change in free-energy when the pressure is varied. When we alter the pressure, the position of the top of the barrier, denoted by $n^{*}$, can change. However, at the top of the barrier, the partial derivative of the free-energy $G$ with respect to size $n$, $\partial G / \partial n$, is zero, so the last term in Eq. (10) drops out. In the metastable vapor, $n=0$ and remains zero, so for the vapor phase the last term is also zero. So we have for the top of the barrier

$$
d G\left(n^{*}\right)=V\left(n^{*}\right) d P,
$$

and, similarly, for the vapor phase,

$$
d G(0)=V(0) d P,
$$

from which we obtain for the variation of the barrier height $\Delta G^{*}$ with pressure

$$
d \Delta G^{*}=d\left[G\left(n^{*}\right)-G(0)\right]=\left[V\left(n^{*}\right)-V(0)\right] d P .
$$

The height of the free-energy barrier at a pressure $P$ can now be obtained by taking the height of the barrier at the reference pressure $P_{\text {ref }}$, as obtained by the umbrella-sampling technique, and by integrating Eq. (13):

$$
\Delta G^{*}(P)=\Delta G^{*}\left(P_{\mathrm{ref}}\right)+\int_{P_{\mathrm{ref}}}^{P}\left[V\left(n^{*}\right)-V(0)\right] d P^{\prime} .
$$

In order to obtain a good measure for the volume at the top of the barrier, we have to determine the top of the barrier with a high accuracy, as the volume of the system strongly depends on the value of the order parameter, i.e., the size of the largest cluster. We therefore performed, for every pressure, not one but three simulations near the top of the barrier: one at the estimated top, and one at each side. The clustersize probability distribution functions of these three simulations were fitted to a polynomial to get the relative freeenergies [via Eq. (7)] of the droplets in the vicinity of the top of the barrier. From this we could then deduce the position of the top of the barrier. Finally, to obtain the volume at the top of the barrier, the value of the critical droplet size was inserted into the expression for the volume as a function of droplet size, which was obtained by fitting the volume histograms to a polynomial.

\section{TOLMAN LENGTH AND SURFACE OF TENSION}

The surface of a droplet in the vapor is not sharp. Rather, it is a transition layer of physical inhomogeneity in which the properties of the fluid change smoothly. However, it is convenient to treat the actual droplet in the vapor as being uniform up to an imaginary surface of zero thickness, the socalled dividing surface, which separates the droplet from the (uniform) vapor.

The position of this dividing surface can be obtained via a thermodynamic route and via a mechanical route. However, both approaches are not equivalent. Below, we indicate how we have computed both the thermodynamically and mechanically defined surface tensions and surfaces of tension. In Sec. VIE we show that the mechanical route cannot be used to compute the height of the nucleation barrier.

\section{A. Thermodynamic description}

In the thermodynamic approach, introduced by Gibbs, ${ }^{48}$ one can derive the generalized Laplace equation, ${ }^{30,31}$ which relates the pressure difference over the drop $\Delta p$ to the location, $R$, of the dividing surface, and to the surface tension $\gamma$.

$$
\Delta p=\frac{2 \gamma(R)}{R}+\frac{\partial \gamma(R)}{\partial R} .
$$

Here, $\Delta p=p_{l}-p_{v}$, with $p_{l}$ and $p_{v}$ the pressure in the liquid and vapor region, respectively. Of course, the pressure in the vapor is always well defined and is equal to the pressure of a homogeneous (bulk) vapor phase with the same density as the density in the vapor region far away from the drop. When the droplet is large enough, the pressure inside the drop is then also well defined and equal to that of a bulk liquid phase with a density which equals that of the density in the core of the drop. However, for smaller droplets the pressure in the core may differ from the bulk liquid pressure. Yet it is important to realize that the generalized Laplace relation is derived from the hypothetical model system, in which the actual droplet is replaced by a droplet that has bulk properties; i.e., it is uniform in density and pressure up to the dividing surface. Therefore, the pressure $p_{l}$ in the drop is that of a hypothetical bulk liquid phase which has a chemical potential $\mu_{l}\left(p_{l}, T\right)$ equal to the chemical potential $\mu_{v}\left(p_{v}, T\right)$ of the (bulk) vapor phase at pressure $p_{v}$.

The thermodynamically defined surface of tension is the surface for which the second term on the right-hand side of Eq. (15) vanishes. Another natural choice for the dividing surface is the so-called equimolar dividing surface, which is defined as the surface for which the excess number of particles at the surface is zero. The Tolman length ${ }^{29} \delta_{T}$ is usually defined as the difference $\delta$ between these two dividing surfaces in the planar limit

$$
\delta_{T} \equiv \lim _{R_{e}, R_{s} \rightarrow \infty} \delta=\lim _{R_{e}, R_{s} \rightarrow \infty}\left(R_{e}-R_{s}\right),
$$

where $R_{e}$ is the radius of the equimolar dividing surface and $R_{s}$ is the radius of the surface of tension.

For any choice of the dividing surface, the (Gibbs) freeenergy of a droplet with radius $R$ is given by ${ }^{30}$

$$
\Delta G^{*}=-\frac{4}{3} \pi R^{3} \Delta p+4 \pi R^{2} \gamma(R),
$$

where $\Delta p$ is given by the generalized Laplace equation, i.e., Eq. (15). If we take for the dividing surface the surface of tension, then Eq. (17) reduces to

$$
\Delta G^{*}=\frac{4}{3} \pi R_{s}^{2} \gamma_{s}
$$

or, equivalently,

$$
\Delta G^{*}=\frac{2}{3} \pi \Delta p R_{s}^{3} .
$$

We have used the above equation, with $\Delta G^{*}$ and $\Delta p$ obtained from the simulations, to compute the thermodynamical surface tension and surface of tension.

\section{B. Mechanical description}

The thermodynamic description of droplets is macroscopic in nature. In order to establish a link with molecular 
properties, it would seem more natural to use a mechanical picture. This approach would allow one to relate the surface tension and surface of tension of a droplet to microscopic quantities, such as the pressure tensor. However, the mechanical and thermodynamic definitions are not equivalent. As a result, the surface tension and dividing surface that are computed mechanically cannot be used to predict the height of the nucleation barrier. In fact, our simulations show that the mechanical route may lead to unphysical results. Below we discuss the mechanical description.

By considering the force and moment acting on a hypothetical strip cutting the surface of the drop, Buff showed that is possible to obtain a mechanical definition of the surface tension. ${ }^{30,31,49}$ The position, $R_{s, m}$, of the surface of tension is given by

$$
R_{s, m}=\frac{\int_{0}^{\infty}\left[p_{l v}\left(r ; R_{s, m}\right)-p_{T}(r)\right] r^{2} d r}{\int_{0}^{\infty}\left[p_{l v}\left(r ; R_{s, m}\right)-p_{T}(r)\right] r d r}
$$

and the surface tension, $\gamma_{s, m}$, acting on the surface of tension is given by

$$
\gamma_{s, m}=\frac{1}{R_{s, m}} \int_{0}^{\infty}\left[p_{l v}\left(r ; R_{s, m}\right)-p_{T}(r)\right] r d r
$$

and also by

$$
\gamma_{s, m}=\frac{1}{R_{s, m}^{2}} \int_{0}^{\infty}\left[p_{l v}\left(r ; R_{s, m}\right)-p_{T}(r)\right] r^{2} d r .
$$

In the above equations, $p_{T}(r)$ is the tangential pressure and $p_{l v}$ is a step function, such that $p_{l v}\left(r ; R_{s, m}\right)=p_{l}^{\prime}$ for $r$ $<R_{s, m}$ and $p_{l v}\left(r ; R_{s, m}\right)=p_{v}$ for $r>R_{s, m}$. The subscript $m$ indicates that we consider the mechanical surface of tension. There are two logical choices for $p_{l}^{\prime}$ : the first is the one introduced below Eq. (15), namely the pressure of the bulk liquid at the chemical potential of the vapor. The second is the actual, local pressure in the core of the droplet. For some of the relations that we will employ, we can choose either definition. However, as we indicate below, in some equations the choice is not free-only the second definition can be used.

From the hydrostatic equilibrium condition $\nabla \cdot \mathbf{p}=0$ we obtain $^{30,31}$

$r_{v}^{n} p_{N}\left(r_{v}\right)-r_{l}^{n} p_{N}\left(r_{l}\right)=\int_{r_{l}}^{r_{v}}\left[(n-2) p_{N}(r)+2 p_{T}(r)\right] r^{n-1} d r$,

where $p_{N}(r)$ is the normal component of the pressure tensor, $r_{v}$ denotes a position far away from the drop in the vapor, and $r_{l}$ is a position in the drop. Integrating the step-function $p_{l v}(r ; R *)$ yields

$$
\int_{r_{l}}^{r_{v}} p^{l v}\left(r ; R^{*}\right) n r^{n-1} d r=r_{v}^{n} p_{v}-r_{l}^{n} p_{l}^{\prime}+R^{* n}\left(p_{l}^{\prime}-p_{v}\right) .
$$

Far away from the drop the local pressure is equal to the vapor pressure, i.e., $p_{N}\left(r_{v}\right)=p_{T}\left(r_{v}\right)=p_{v}$. Furthermore, if we take $r_{l}$ at the center of the drop, i.e., $r_{l}=0$, then Eq. (23) can be subtracted from Eq. (24) to give

$$
\begin{aligned}
p_{l}^{\prime}-p_{v}= & \frac{1}{R^{* n}} \int_{0}^{r_{v}}\left\{n\left[p^{l v}\left(r ; R^{*}\right)-p_{N}(r)\right]\right. \\
& \left.+2\left[p_{N}(r)-p_{T}(r)\right]\right\} r^{n-1} d r .
\end{aligned}
$$

The actual value of $p_{N}\left(r_{l}\right)$ drops out of the above equation provided that $r_{l}=0$ and $n \neq 0$. Under those conditions we are free to choose our definition of $p_{l}^{\prime}$. However, for $n=0$ the choice of $p_{l}^{\prime}$ is no longer free. In that case, we must take $p_{l}^{\prime}$ equal to the normal component of the local pressure in the center of the droplet, i.e., $p_{l}^{\prime}=p_{N}(0)$. Note that with this choice for $p_{l}^{\prime}$, we need not choose $r_{l}=0$ to arrive at Eq. (25). In the following, we therefore take $p_{l}^{\prime}$ to be the local pressure in the center of the droplet. We stress that, except for very large droplets, this definition differs from the thermodynamic one.

For $n=2 \mathrm{Eq}$. (25) reduces to

$$
p_{l}^{\prime}-p_{v}=\frac{2}{R^{* 2}} \int_{0}^{\infty}\left[p_{l v}\left(r ; R^{*}\right)-p_{T}(r)\right] r d r .
$$

This relation is valid for any position of the dividing surface. If we position the dividing surface at the thermodynamic surface of tension with radius $R_{s}$, we obtain

$$
p_{l}^{\prime}-p_{v}=\frac{2}{R_{s}^{2}} \int_{0}^{\infty}\left[p_{l v}\left(r ; R_{s}\right)-p_{T}(r)\right] r d r .
$$

If the core of the droplet behaves as a bulk liquid, that is, if the actual pressure $p_{l}^{\prime}$ in the droplet is equal to the pressure $p_{l}$ of a bulk liquid with a chemical potential that is equal to that of the vapor phase, then we can combine the above equation with the Laplace equation, Eq. (15), to arrive at

$$
\gamma_{s}=\frac{1}{R_{s}} \int_{0}^{\infty}\left[p_{l v}\left(r ; R_{s}\right)-p_{T}(r)\right] r d r .
$$

This equation has the same structure as Eq. (21). However, this does not imply that the thermodynamic and mechanical surface tensions and surfaces of tensions are equal. In fact, Blokhuis et al. ${ }^{19}$ have shown that the positions of the surfaces can differ significantly, and our simulations show that in fact they do.

It is possible to relate $R_{s, m}$ and $\gamma_{s, m}$ to $p_{l}^{\prime}-p_{v}$ in an expression, which, to increase the confusion, looks like the Laplace relation. We take $R^{*}=R_{s, m}$ in Eq. (26) and combine the resulting expression with Eq. (21), to arrive at

$$
p_{l}^{\prime}-p_{v}=\frac{2 \gamma_{s, m}}{R_{s, m}} .
$$

So also for the mechanically defined surface tension and surface of tension, a Laplace-type relation is fulfilled. We can now exploit this relation to express $R_{s, m}$ and $\gamma_{s, m}$ in terms of $p_{N}(r)-p_{v}$, from which we can compute the mechanically defined surface tension and surface of tension. We start by 
combining the above equation and Eq. (25) with $n=3$ and with $R^{*}=R_{s, m}$, and subtract the resulting expression from Eq. (22), to obtain

$$
\int_{0}^{r_{v}}\left[p^{l v}\left(r ; R_{s, m}\right)-p_{N}(r)\right] r^{2} d r=0 .
$$

This equation can be combined with Eq. (22) to yield

$$
\gamma_{s, m}=\frac{1}{R_{s, m}^{2}} \int_{0}^{\infty}\left[p_{N}(r)-p_{T}(r)\right] r^{2} d r .
$$

In Refs. 30 and 31 it is suggested that a similar expression can be derived for the thermodynamically defined surface tension by combining the expression following from Eq. (25), with $n=3$ and $R=R_{s}$, and the generalized Laplace equation [Eq. (15)] with Eq. (22). However, in order to arrive at this result, one has to make the assumption that the mechanical and thermodynamic descriptions are equivalent.

We do not make the identification between the thermodynamic and mechanical descriptions. Instead, we make use of the fact that for the mechanically defined surface of tension a Laplace-type relation also holds, and use the relations that follow from the hydrostatic equilibrium condition for this dividing surface. For instance, it is possible to obtain another expression for the mechanically defined surface tension by combining Eq. (29) with Eq. (25) for $n=0$ and $R^{*}$ $=R_{s, m}$

$$
\gamma_{s, m}=R_{s, m} \int_{0}^{\infty}\left[p_{N}(r)-p_{T}(r)\right] r^{-1} d r .
$$

We can obtain the position of the mechanical surface of tension by combining the above equation with Eq. (31)

$$
R_{s, m}^{3}=\frac{\int_{0}^{\infty}\left[p_{N}(r)-p_{T}(r)\right] r^{2} d r}{\int_{0}^{\infty}\left[p_{N}(r)-p_{T}(r)\right] r^{-1} d r} .
$$

The above three equations can be rewritten using

$$
p_{T}(r)=p_{N}(r)+\frac{r}{2} \frac{d p_{N}(r)}{d r},
$$

which follows from the condition of hydrodynamic equilibrium, $\nabla \cdot \mathbf{p}=0$. We then obtain

$$
\begin{aligned}
& R_{s, m}^{3}=\frac{3}{\Delta p} \int_{0}^{\infty}\left[p_{N}(r)-p_{v}\right] r^{2} d r, \\
& \gamma_{s, m}^{3}=\frac{3}{8} \Delta p^{2} \int_{0}^{\infty}\left[p_{N}(r)-p_{v}\right] r^{2} d r,
\end{aligned}
$$

where $\Delta p=p_{l}^{\prime}-p_{v}$. We have used these equations to compute the mechanically defined surface tensions and surfaces of tension.

In the thermodynamic model, the height of the barrier is given by Eq. (19). It is clear that if the mechanical and thermodynamic descriptions were equivalent, combining Eq. (19) with Eq. (35) would yield a microscopic expression for the height of the barrier [Eq. (37), see Refs. 17 and 50].
However, as the mechanical and thermodynamic pictures cannot be mixed, the height of the barrier cannot be expressed in terms of an integral of the pressure profile,

$$
\Delta G^{*} \neq 2 \pi \int_{0}^{\infty}\left[p_{N}(r)-p_{v}\right] r^{2} d r .
$$

Figure 5 illustrates the kind of errors that may result if the mechanical and thermodynamic pictures are confused.

We stress that the distinction between mechanical and thermodynamic expression for the surface tension should not be confused with the ambiguity in the definition of the microscopic stress tensor. Schofield and Henderson ${ }^{51}$ have proposed a general expression for the stress tensor. This expression reflects the freedom that we have in defining the momentum flux density in a fluid. The commonly used Irving-Kirkwood pressure tensor, ${ }^{52}$ and the Harasima pressure tensor, ${ }^{24,51,53,54}$ are special cases of the SchofieldHenderson expression. Both from simulations ${ }^{54}$ and from theory, ${ }^{51}$ these expressions are known to give the same surface tension for a planar interface, as the surface tension is related to the zeroth moment of $p_{N}(r)-p_{T}(r)$. However, the position of the mechanical surface of tension, which is related to the first moment, is not insensitive to the choice of the pressure tensor. The Irving-Kirkwood expression appears to be the most natural choice, as the contour joining two interacting particles, which determines where the force is acting, corresponds to a straight line. Furthermore, Blokhuis et al. ${ }^{19}$ showed that the Irving-Kirkwood pressure tensor, in contrast to the Harasima pressure tensor, leads to expressions for the pressure difference, the surface tension, and the Tolman length, that agree with expressions found using microscopic sum rules. We have therefore used the Irving-Kirkwood expression for the pressure tensor to compute the pressure profiles.

\section{COMPUTATIONAL DETAILS}

We have studied homogeneous gas-liquid nucleation in a Lennard-Jones system in which the interaction potential was truncated and shifted at a cutoff radius $r_{c}=2.5 \sigma$, where $\sigma$ is the particle diameter. We made no long-range corrections and applied cubic periodic boundary conditions. In the following, we use reduced units, such that the Lennard-Jones well depth $\epsilon$ is the unit of energy, while the Lennard-Jones diameter $\sigma$ is the unit of length.

In most experimental studies of homogeneous nucleation, the volume is fixed and nucleation of liquid droplets from the vapor phase leads to a decrease of the vapor pressure. However, as the concentration of nuclei is very small, the drop in vapor pressure is negligible and the pressure and chemical potential effectively remain constant during the nucleation process. In principle, we could simulate the experimental situation by performing a $N V T$ simulation. A large excess number of vapor particles would then be needed in order to keep the vapor pressure constant, which can be achieved by simulating a very large system. However, this would make the simulations unnecessarily expensive. It is much more natural to work in the $N P T$ or in the $\mu V T^{55}$ ensemble. In the $N P T$ ensemble, as the droplet is formed, the 


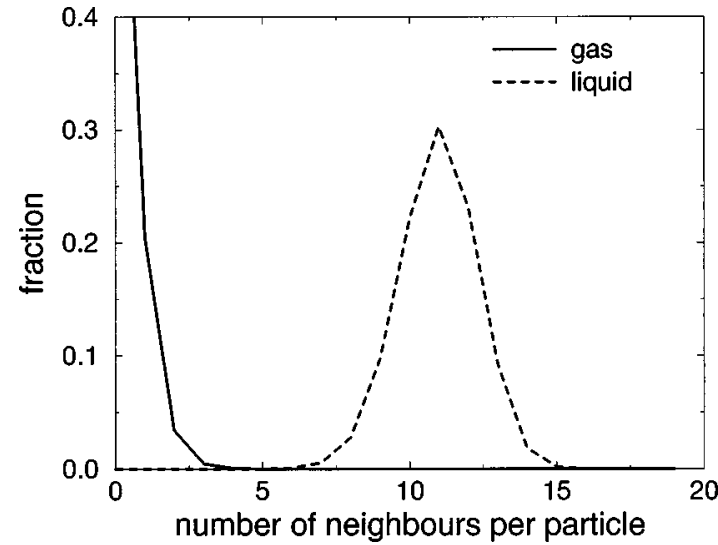

FIG. 1. Distributions of the number of neighbors per particle, denoted by $N C P$, in a Lennard-Jones system for a thermally equilibrated liquid and vapor, at coexistence ( $T=0.741, P=0.00783$ ). Two particles are considered to be neighbors if they are separated by a distance less than $q_{c}=1.5$.

volume is adjusted such that the pressure (and the chemical potential) in the vapor will remain constant. In this ensemble it is therefore not necessary to simulate a large number of vapor particles. The same holds for the $\mu V T$ ensemble. However, in this approach insertions and removals of particles from the system are required, which can be inefficient or even impossible at high densities. We have chosen to work in the NPT ensemble.

In our simulations, we need an operational definition of liquid-like clusters. We do this by making a distinction between particles that have a liquid-like and a vapor-like environment. Liquid-like particles are particles that experience a local density that is significantly higher than that of the vapor. There is no unique definition of the local density surrounding a particle. In the following, we use the number of particles within a spherical shell of radius $q_{c}$ as a measure of the local density. The distribution functions of the number of neighbors per particle in the liquid and in the vapor are shown in Fig. 1. Note that the distribution functions hardly overlap. We have therefore adopted the criterion that all particles which have more than four neighbors are considered to be liquid-like.

After we have identified which particles in the system are liquid-like, we can determine the liquid-like clusters. We have applied the criterion that any two liquid-like particles which have a distance less than $q_{c}=1.5$ (which corresponds to the first minimum in the radial distribution function of the liquid) belong to the same liquid cluster. Note that our definition of a cluster is close to the one used by Stillinger, ${ }^{37}$ but not quite equivalent. Stillinger adopted the criterion that any two particles that are within a certain cutoff distance belong to the same cluster. So even particles that, in our definition are considered to be vapor particles, can be part of a liquid cluster according to Stillinger's criterion.

The nucleation barrier can be measured both by MC and MD. The advantage of MD is that it gives faster equilibration of pressure gradients through collective particle motions. However, with MD the diffusion of the system through the order-parameter window in the umbrella-sampling simulations is rather slow. The density in the vapor is very low and in MD both the liquid and vapor particles move on the same time scale. Therefore, in MD the fluctuations in the size of the largest cluster, i.e., the order parameter, are limited by the influx of particles from the vapor.

The advantage of MC is that it is particularly suited for the umbrella-sampling scheme as, in contrast to MD, the forces associated with the biasing potential do not have to be calculated. Furthermore, one can perform tricks to facilitate the sampling of configuration space. In order to speed up the diffusion of particles in the vapor, the particles in the vapor phase are given a different maximum displacement from the particles in the liquid phase. Of course, this introduces a bias which needs to be corrected in order to satisfy detailed balance. This is described in Appendix B.

We have tested both MD and MC by determining the "diffusion constant" of the order parameter at the top of the barrier. It was found that per MD timestep or per MC cycle (in which, on average, every particle is given one trial displacement)

$$
\sqrt{\frac{\left\langle\Delta n^{2}\right\rangle_{\mathrm{MC}}}{\left\langle\Delta n^{2}\right\rangle_{\mathrm{MD}}}} \approx 7-8
$$

However, the number of cycles performed per unit of CPU time was three times higher for MD, so that the effective diffusion constant for MC was about two to three times that of MD. We did use MD to speed up the equilibration, but most of the actual simulations were performed using $\mathrm{MC}$ sampling.

In the umbrella-sampling scheme, the system should sample configuration space according to the potential

$$
U_{i}\left(\mathbf{r}^{N}\right)=U_{0}\left(\mathbf{r}^{N}\right)+W\left[n\left(\mathbf{r}^{N}\right)\right],
$$

where $U_{0}\left(\mathbf{r}^{N}\right)$ is the potential of the original model system and $W\left[n\left(\mathbf{r}^{N}\right)\right]$ is the biasing potential as defined in Eq. (8). In principle, we could recompute the size of the largest cluster and the biasing potential after every MD or MC cycle, or even after every particle displacement in the MC simulations. However, this would be far too time consuming. We therefore adopted a staged scheme. In the first stage, a series of MD or MC cycles is performed without the biasing potential. In the second stage, after the unbiased trajectory, the size of the cluster and the biasing potential are recalculated. To ensure that configuration space will be sampled in accordance with the potential in Eq. (39), the trajectory is then accepted with a probability which is determined by $\exp \left[-\beta \Delta W\left[n\left(\mathbf{r}^{N}\right)\right]\right]$, where $\Delta W\left[n\left(\mathbf{r}^{N}\right)\right]$ is the difference in biasing potential before and after the trajectory.

In the MC simulations, each trial move consisted either of an attempted particle displacement or a trial volume change. The choice between trial particle moves and trial volume moves was made at random. As we used a fixed cutoff in real coordinates for the intermolecular interactions, the potential energy did not scale with a volume move, and we had to recalculate the total potential energy from scratch after every trial volume move. Therefore, an MC cycle consisted on average of only one trial volume move and one trial displacement per particle. The acceptance ratio for both types of MC moves was kept at $50 \%$ by adjusting the maxi- 
mum size of the move. The maximum displacements for the liquid and vapor particles were tuned independently.

The length of the trajectories depends on the computational cost of evaluating the order parameter and the average probability with which the trajectories are accepted. To be more precise, the efficiency $\nu$ is proportional to

$$
\nu \propto \frac{P_{\text {acc }}(l)}{a+b / l} .
$$

Here, $l$ is the length of the trajectories and $a$ and $b$ denote the computational costs of performing an $\mathrm{MD} / \mathrm{MC}$ cycle and a cluster analysis, respectively. $P_{\text {acc }}(l)$ is the acceptance probability of the trajectories, which depends on the trajectory length and on the force constant of the biasing potential (as well as on the steepness of the underlying free-energy barrier). Typically, for $k_{n}=0.01-0.02$, a trajectory length of 50 MC cycles was optimal. To speed up the simulations, we used both a linked list and a neighbor list for the calculation of the energies and the identification of the clusters. ${ }^{56}$

The number of umbrella windows for the free-energy barrier was 15. A typical simulation in a window consisted of an equilibration period of 100 000-250 000 MD steps, followed by a production run of $250000 \mathrm{MC}$ cycles. The individual probability distribution functions $P(n)$ obtained in the different runs were fitted simultaneously to a polynomial. We used a polynomial fit rather than the self-consistent scheme of Ferrenberg and Swendsen, ${ }^{57}$ because not all adjacent histograms overlapped.

\section{RESULTS AND DISCUSSION}

We studied the nucleation of liquid droplets from the vapor as a function of supersaturation. All simulations were performed for one temperature, $T=0.741$, which is $32 \%$ below the critical temperature $\left(T_{c}=1.085\right) .{ }^{58}$ At this temperature, the pressure and densities of the coexisting phases are known. ${ }^{58}$ Furthermore, in order to make a comparison with nucleation theories, we have to know the surface tension. The temperature of our simulations is in the range of temperatures for which Chapela et al. ${ }^{59}$ and, more recently, Holcomb et al. ${ }^{60}$ computed surface tensions for planar gasliquid interfaces.

The number of particles was $N=864$. As the size of the largest critical nucleus obtained in the simulations is around 300 particles, corresponding to the smallest degree of supersaturation, and, more importantly, the density in the surrounding vapor is very low, with this number of particles the size of the simulation box was always large enough compared to the size of the critical droplets for system-size effects not to be present.

\section{A. The nucleation barrier}

We first computed the full nucleation barrier with the umbrella-sampling technique for a reference pressure. This reference pressure was chosen to be $P=0.01202$, which corresponds to a supersaturation $S=P / P_{\text {coex }}=1.53$. Figure 2 shows the nucleation barrier for this degree of supersaturation.

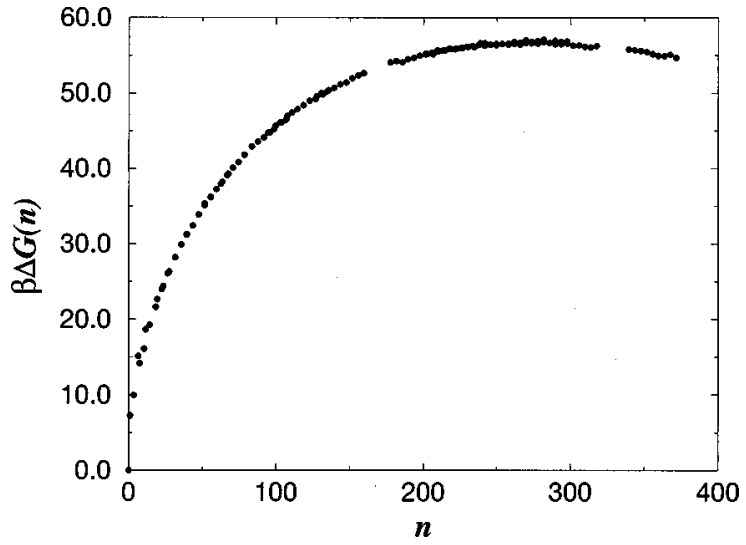

FIG. 2. The Gibbs free-energy barrier as a function of the size of a cluster, denoted by $n$, at a supersaturation of $S=1.53(T=0.741, P=0.01202)$.

In our simulations we are able to study not only critical droplets, but also pre- and postcritical droplets. Visual inspection revealed that precritical droplets consisting of only 10-25 particles are already quite spherical. We therefore computed the density as a function of $r$, the distance to the center-of-mass of the cluster. Figure 3 shows the radial density profiles for several precritical and critical clusters. For the smallest droplets, the density already approaches a bulk liquid density in the core. We stress that this is not due to our choice of the cluster definition, which requires that a cluster particle should have at least five neighbors. A radial profile of the number of connections per particle shows that, in the core of the liquid droplet, the number of connections per particle is much larger than the threshold value of our cluster definition. In fact, it also approaches a bulk liquid value, i.e., around 12 neighbors per particle. Hence, our results are not very sensitive to the choice of the threshold value.

Figure 3 also shows that the density in the core hardly increases when the cluster grows to its critical size $n^{*}$ $=280$. Furthermore, the density profiles show that the width

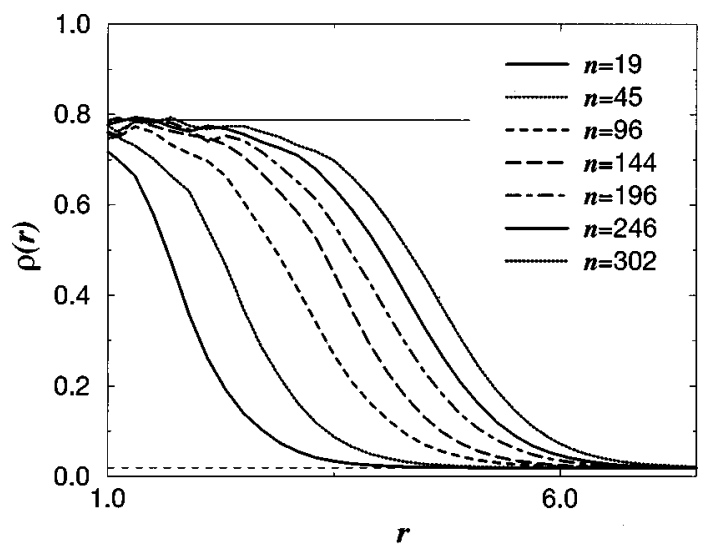

FIG. 3. Density as a function of $r$, the distance to the center-of-mass of the clusters, for several precritical and critical nuclei at a supersaturation of $S$ $=1.53(T=0.741, P=0.01202)$. The thin horizontal dashed line denotes the density in the vapor, and the thin horizontal solid line denotes the density in the bulk liquid, which has a chemical potential equal to that of the vapor phase. 


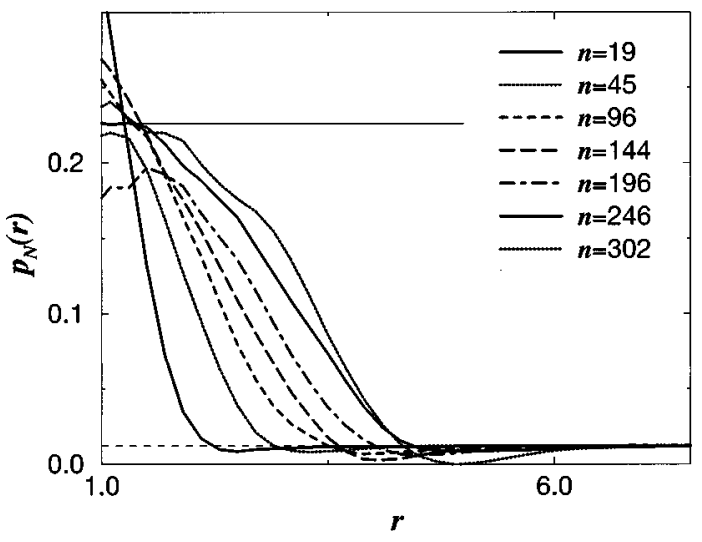

FIG. 4. Radial profiles of the normal component of the pressure tensor, $p_{N}(r)$, for several precritical and critical nuclei at a supersaturation of $S$ $=1.53(T=0.741, P=0.01202)$. The thin horizontal dashed line denotes the pressure in the bulk vapor, and the thin horizontal solid line denotes the pressure in the bulk liquid, which has a chemical potential equal to that of the vapor phase.

of the interface remains essentially constant at approximately $3.5 \sigma$.

Using the Irving-Kirkwood expression for the pressure tensor, ${ }^{52}$ we also computed the pressure profiles for the precritical and critical nuclei. In Fig. 4, we show the radial profiles of the normal component of the pressure tensor. It is seen that all pressure profiles smoothly go to a bulk vapor value for large values of $r$, i.e., far away from the center of the droplet. In the center of the droplet, the statistical accuracy with which the pressure can be determined is low, as the volume is small. Nevertheless, the data suggests that, except for the smallest droplets, the pressure profiles approach a plateau value in the core. In fact, the pressure in the core, as well as the density (see Fig. 3), approaches that of a hypothetical bulk liquid at a chemical potential that is equal to the chemical potential in the vapor phase. This indicates that the interior of the droplets shows bulk liquid-like behavior.

From the normal component of the pressure tensor we can also obtain the transverse component of the pressure tensor using Eq. (34). This equation shows that, when the derivative of the normal component of the pressure tensor with respect to the radius is positive, the transverse component is larger than the normal component of the pressure tensor. Under these conditions the surface would be under compression, rather than under tension. When the normal component is larger than the transverse component, the surface is under tension.

Figure 4 shows that most of the surface of the droplets is under tension. However, at the vapor side of the interface the normal pressure becomes smaller than the vapor pressure. As the profile subsequently approaches the bulk vapor value, it is clear that there is a small region in which the derivative of the normal-pressure profile is positive. In this region the interface is under compression, rather than under tension. This behavior is also found in theoretical calculations, for different potentials, ${ }^{61-63}$ as well as in a computer-simulation study of liquid droplets by Thompson et al., ${ }^{24}$ and in the computer simulations of a flat interface by Walton et al. ${ }^{54}$

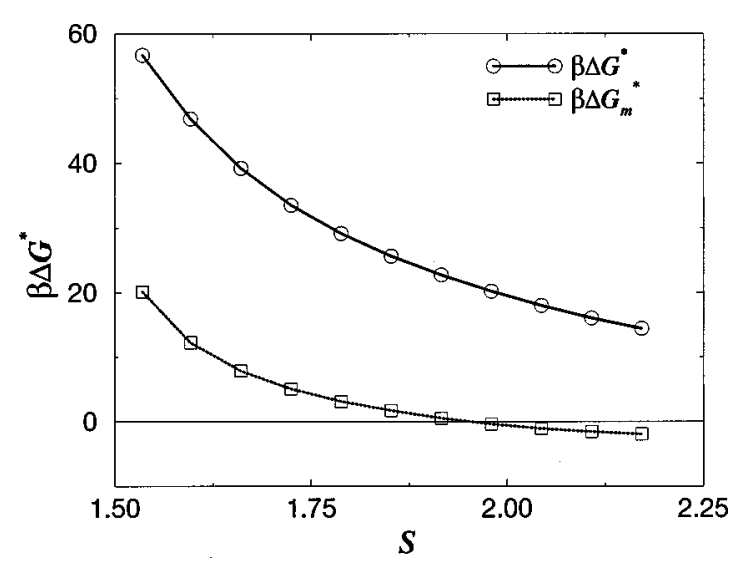

FIG. 5. The height of the nucleation barrier as a function of supersaturation $S$ for $T=0.741$. The solid line is obtained via a thermodynamic integration technique, see Eq. (14). The dashed line is obtained by integrating the pressure profile, see Eq. (37). It is seen that the mechanical route to the nucleation barrier (dashed line) does not give the correct height of the free-energy barrier.

\section{B. Dependence on supersaturation}

Using the method described in Sec. III B, we computed the height of the nucleation barrier as a function of supersaturation. Figure 5 shows the free-energy barrier as a function of supersaturation. We could not continue our simulations beyond $S=2.2$, because at this point the height of the barrier is so low that spontaneous nucleation of additional droplets occurs in the vapor. But, before we discuss the height of the nucleation barrier as a function of supersaturation in more detail, let us first describe qualitatively how the critical nucleus changes as the supersaturation is increased.

In Figs. 6 and 7 we show, respectively, the density and pressure profiles for three critical nucleus sizes. In the legends, we have also indicated the excess number of particles defined as

$$
\Delta n=4 \pi \int_{0}^{\infty}\left[\rho(r)-\rho_{v}\right] r^{2} d r
$$

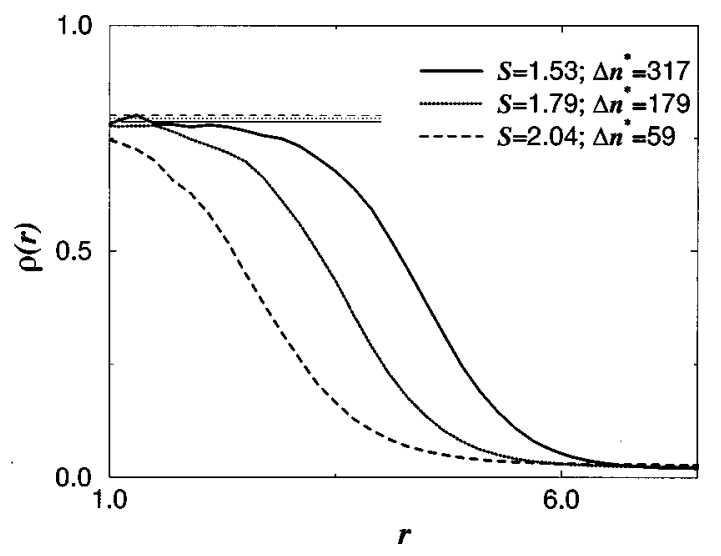

FIG. 6. Radial density profiles for different critical nuclei, at different supersaturations $S(T=0.741)$. In the legends, the excess number of particles, $\Delta n^{*}$, is also indicated. The thin horizontal lines indicate, for the different supersaturations, the density in the bulk liquid that has the same chemical potential as the vapor phase. 


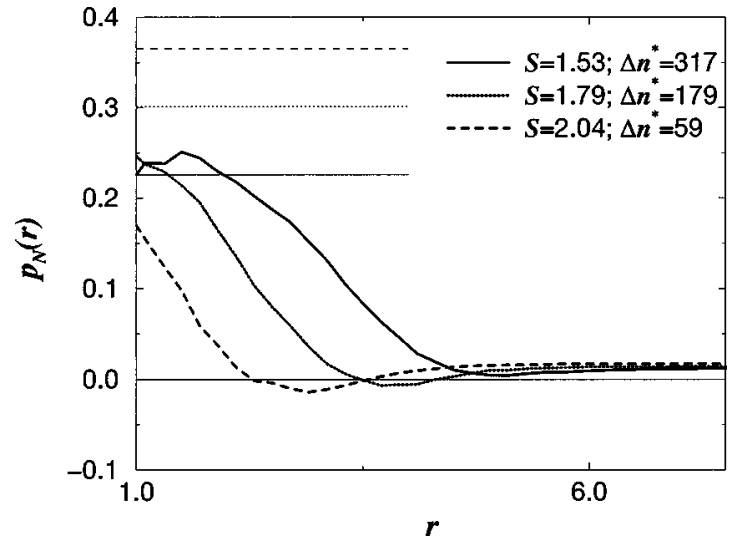

FIG. 7. Radial profiles of the normal component of the pressure tensor for critical nuclei, at different supersaturations $S(T=0.741)$. The excess number of particles $\Delta n^{*}$ is also given. The thin horizontal lines indicate, for the different supersaturations, the pressure in the bulk liquid that has the same chemical potential as the vapor phase.

where $\rho_{v}$ is the density in the vapor (far away from the droplet). Because of the diffuse nature of the interface, the size of the critical nucleus is ambiguous and depends on the position of the dividing surface. However, the excess number of particles is independent of the position of the dividing surface and is therefore a more meaningful quantity.

Figures 6 and 7 show that as the supersaturation is increased, the density and pressure in the core slowly decrease. This indicates that the droplet already starts to lose bulk behavior in the core. If the core of the droplet showed bulk liquid behavior, then, in order for the chemical potential in the bulk liquid core to be equal to the chemical potential in the vapor, the density and pressure in the core of the droplet would have to increase when the pressure in the vapor increased. Indeed, this behavior has been observed by Thompson $e$ t al. ${ }^{24}$ and Nijmeijer et $a l .{ }^{26}$ in their simulations of liquid droplets. However, the droplets for which this behavior was seen were much larger (larger than 2000 particles) than the droplets studied here. In fact, in the simulations of Thompson et al. ${ }^{24}$ a clear crossover in behavior was seen for smaller droplets. In line with the present results, they found that once the droplets are smaller than a certain size, the density and pressure in the core of the droplets decrease with increasing vapor pressure. This loss of bulk behavior is also found in several molecular theories. ${ }^{16,61-63}$

Figures 6 and 7 suggest that the width of the interface remains constant over the range of droplet sizes studied. Note that when the droplets become smaller, the region for which the normal pressure is smaller than the vapor pressure increases. Moreover, the region for which $p_{T}>p_{N}$ increases as well; that is, the interface is progressively under more compression. In fact, for the smallest droplets studied, most of the interface is under compression rather than tension. This trend has also been observed by Falls et al. ${ }^{61}$

\section{The nucleation theorem}

The nucleation theorem is a powerful tool to analyze experimental data on homogeneous nucleation., ${ }^{3,8-10}$ The nucleation theorem states that the excess number of mol- ecules of a given component in the critical nucleus can be obtained from the variation of the height of the barrier with the chemical potential, $\mu_{v, i}$, of that component in the vapor phase:

$$
\frac{\partial \Delta G^{*}}{\partial \mu_{v, i}}=-\Delta n_{i}^{*},
$$

where $\Delta n_{i}^{*}$ is defined as in Eq. (41) above.

As nucleation is a rare event, it is impossible to measure the size and composition of critical nuclei directly in an experiment. However, if one assumes that the prefactor in the expression for the nucleation rate depends only weakly on supersaturation, the nucleation theorem makes it possible to determine the size and composition of the critical nuclei as a function of the activities of the components in the vapor phase.

Several derivations of the nucleation theorem have been presented in the literature. The original derivation by Kashchiev ${ }^{5}$ and a later one by Viisanen et al. ${ }^{9}$ were based on a thermodynamic model in which the reversible work of formation of a cluster is written as the sum of a bulk term and an excess free-energy term, which includes contributions from the surface free-energy. The nucleation theorem was obtained by assuming that the variation of the excess freeenergy of the cluster depends only weakly on the chemical potential. However, in the approach of Refs. 5 and 9, the variation of the barrier height with the gas phase activities yields the total number of particles in the cluster, and not the excess number of particles in the cluster. Also, an analysis based on classical nucleation theory by Strey et al. ${ }^{8}$ suggests that, from the variation of the height of the barrier with the chemical potential in the vapor phase, the total number of particles, rather than the excess number of particles, is obtained. However, Oxtoby and Kashchiev, ${ }^{6}$ who also used a thermodynamic approach, showed that the variation of the surface free-energy with the chemical potential in the vapor phase is related to the surface density of the molecules, and that the nucleation theorem gives the excess number of particles and not the total number of particles in the critical cluster. All these derivations are based on thermodynamic models. It is conceivable that such an approach fails for very small droplets. However, Ford ${ }^{7}$ gave a derivation, using small system thermodynamics, which confirmed that the excess number of particles in the cluster is obtained from the variation of the height of the barrier with the gas phase activities. Moreover, the same result was obtained from a statistical mechanical analysis by Viisanen, Strey and Reiss. ${ }^{3}$ In Appendix $\mathrm{C}$, we give a compact derivation which is also based on statistical mechanics. Furthermore, this derivation also shows that the variation of the height of the Gibbs freeenergy barrier with the pressure is related to the excess volume of the system at the top of the barrier, and that the variation of the barrier height (both in the grand-canonical and in the isothermal-isobaric ensemble) with $T$ and $\beta$ gives the excess entropy and excess internal energy, respectively.

In our simulations, we compute the barrier height as a function of pressure. However, for comparison with the nucleation theorem, we need to know the variation of the 


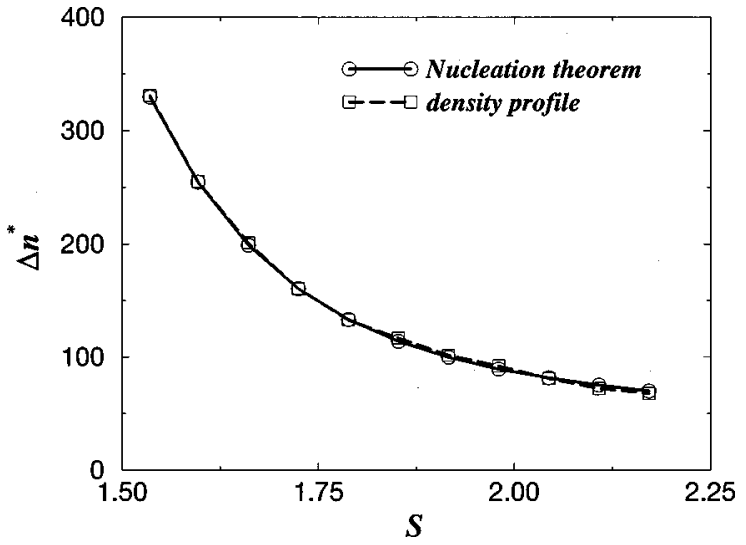

FIG. 8. The excess number of particles $\Delta n^{*}$ in the critical nuclei as a function of the supersaturation $S(T=0.741)$, as obtained from the variation of the height of the barrier with the chemical potential in the vapor phase, and as obtained by integrating the density profiles. According to the nucleation theorem they should give the same results.

barrier height with the chemical potential of the vapor phase. The variation of the chemical potential with pressure is given by the Gibbs-Duhem relation: $d \mu / d P \mid T=1 / \rho_{v}$, where $\rho_{v}$ is the density in the vapor. In Fig. 8 we show the excess number of particles in the cluster obtained via the nucleation theorem and by directly integrating the density profiles. It is seen that for all droplets sizes studied the agreement is excellent. This indicates that the method to measure the height of the barrier as a function of supersaturation described in Sec. III B is not only efficient, but also very accurate.

\section{Deviations from classical nucleation theory}

Recent experiments on gas-liquid nucleation indicate that the sizes of the critical nuclei are accurately predicted by classical nucleation theory., ${ }^{3,10,64}$ However, the rate of nucleation was found to be consistently higher than that predicted by classical nucleation theory. In fact, it was observed that the ratio of the experimentally determined nucleation rate and the nucleation rate as predicted by CNT, although depending on temperature, was nearly independent of the supersaturation. ${ }^{64}$ In two recent papers, McGraw and Laaksonen derived relations for the height of the barrier and the size of the critical nucleus that could provide an explanation for these observations. ${ }^{17,18}$ In their first paper, ${ }^{17}$ they gave a derivation which was based on the nucleation theorem. In the second paper, they gave a derivation which was based on the nonuniform spherical droplet model. ${ }^{18}$ They showed that if the nucleus has an incompressible core with a density equal to that of the bulk phase and the number of particles within the equimolar dividing surface is correctly predicted by $\mathrm{CNT}$, then the difference between the actual barrier height and the height of the barrier as predicted by CNT is independent of the supersaturation and depends only on temperature. Using the techniques discussed in Sec. III B, we are able to test these predictions directly.

In classical nucleation theory, the size of the critical droplet is given by

$$
n^{*}=\frac{32 \pi \gamma_{\infty}^{3}}{3 \rho_{l}^{2} \Delta \mu^{3}},
$$

where $\gamma_{\infty}$ is the surface free-energy of the planar interface and $\rho_{l}$ is the density of the bulk liquid at coexistence. $\Delta \mu$ is the difference between the chemical potential $\mu_{v}$ in the vapor phase and the chemical potential $\mu_{l}$ in the bulk liquid phase, both at the pressure $P$ in the vapor phase, i.e.,

$$
\Delta \mu(P)=\mu_{v}(P)-\mu_{l}(P) .
$$

In classical nucleation theory, the height of the nucleation barrier is given by

$$
\Delta G^{*}=\frac{16 \pi \gamma_{\infty}^{3}}{3 \rho_{l}^{2} \Delta \mu^{2}} .
$$

As the cube of the surface free-energy enters the expressions for the height of the barrier and for the critical nucleus size, we need an accurate estimate for the surface freeenergy. Chapela et al. ${ }^{59}$ and Holcomb et al. ${ }^{60}$ have calculated surface free-energies for a Lennard-Jones system with the same potential cutoff used in our simulations, and for a range of temperatures that encompasses our temperature. In order to obtain the surface free-energy at our temperature, we made a polynomial fit to their data and found $\gamma_{\infty}=0.494$.

The chemical-potential difference $\Delta \mu(P)$ was computed by integrating the difference in the inverse density between the liquid and vapor phase from the coexistence pressure: ${ }^{65}$

$$
\Delta \mu(P)=\int_{P_{\text {coex }}}^{P}\left[\frac{1}{\rho_{v}\left(P^{\prime}\right)}-\frac{1}{\rho_{l}\left(P^{\prime}\right)}\right] d P^{\prime} .
$$

Here, $P_{\text {coex }}$ is the coexisting pressure and $\rho_{v}$ and $\rho_{l}$ are the densities of the bulk vapor and bulk liquid phase, respectively. As the chemical potential of the vapor phase depends very strongly on pressure, the difference in chemical potential is very sensitive to the exact location of the coexistence point. We found that the data of $\mathrm{Smit}^{58}$ was not accurate enough for our purpose, and we therefore performed a more extensive Gibbs ensemble simulation ${ }^{66}$ to calculate the coexistence point. We found that the coexistence pressure $P_{\text {coex }}$ $=0.00783$, and that the density of the liquid at coexistence $\rho_{l}=0.766$.

Figure 9 shows the number of particles in the critical nucleus as a function of $1 / \Delta \mu^{2}$. In the figure, we compare the predictions of CNT and the numerical results for $n_{e}^{*}$, the number of particles within the equimolar dividing surface:

$$
n_{e}^{*}=\frac{4 \pi \rho(0)}{\rho(0)-\rho_{v}} \int_{0}^{\infty}\left[\rho(r)-\rho_{v}\right] r^{2} d r .
$$

Here, $\rho_{v}$ is the density in the vapor, and $\rho(0)$ is the density in the core of the droplet. The statistical error in $\rho(0)$, the density in the core, is relatively large. However, as $\rho_{v} \ll \rho(0)$, this inaccuracy has little effect on the value of $n_{e}^{*}$. For the same reason, $n_{e}^{*}$ is nearly equal to the better defined quantity $\Delta n^{*}$, which is shown in Fig. 9. But more importantly, it is seen that, for all droplet sizes studied, CNT gives a good estimate for the number of particles in the critical cluster. 


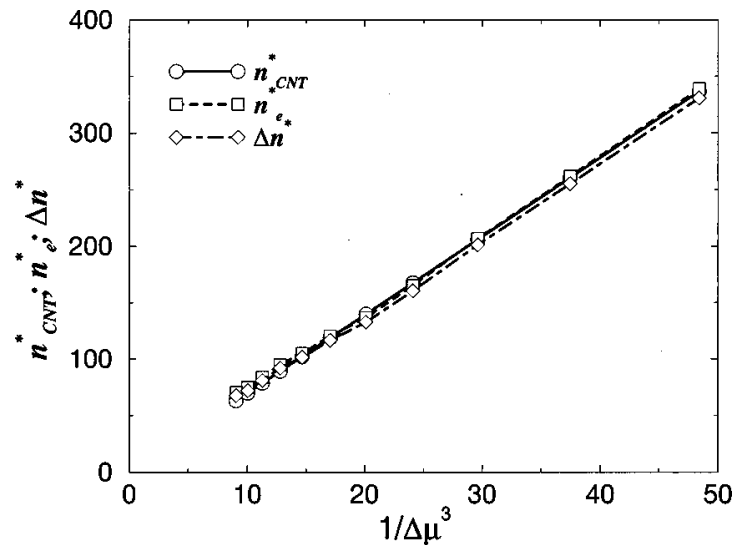

FIG. 9. The number of particles in the critical nuclei as a function of $1 / \Delta \mu^{3}$, as predicted by classical nucleation theory and as obtained from the simulations. The number of particles predicted by classical nucleation theory is indicated by $n_{\mathrm{CNT}}^{*}$, and the number of particles within the equimolar dividing surface, as obtained from the simulations, is indicated by $n_{e}^{*}$. For comparison, we have also shown the excess number of particles, denoted by $\Delta n^{*}$, as obtained from the simulations. According to the relations proposed by McGraw and Laaksonen (Refs. 17, 18), $n_{e}^{*}$ is given by classical nucleation theory $\left(n_{\mathrm{CNT}}^{*}\right)$.

Surprisingly, the predictions of CNT are excellent down to the smallest droplets, which consist almost exclusively of interface.

In Fig. 10, we show that the simulation results support the McGraw-Laaksonen predictions for the height of the barrier: the barrier height found in the simulations differs by a constant offset from the value predicted by CNT. This result appears to hold even for critical nuclei consisting of only $50-100$ particles. Again, this finding is surprising because, as discussed in Sec. VI B, the cores of these nuclei do not show bulk behavior. In fact, McGraw and Laaksonen gave two derivations of their expressions for the height of the nucleation barrier and for the critical nucleus size: one made use of the assumption that the nucleus had an incompressible core, ${ }^{18}$ the other did not. ${ }^{17}$ Our simulations suggest

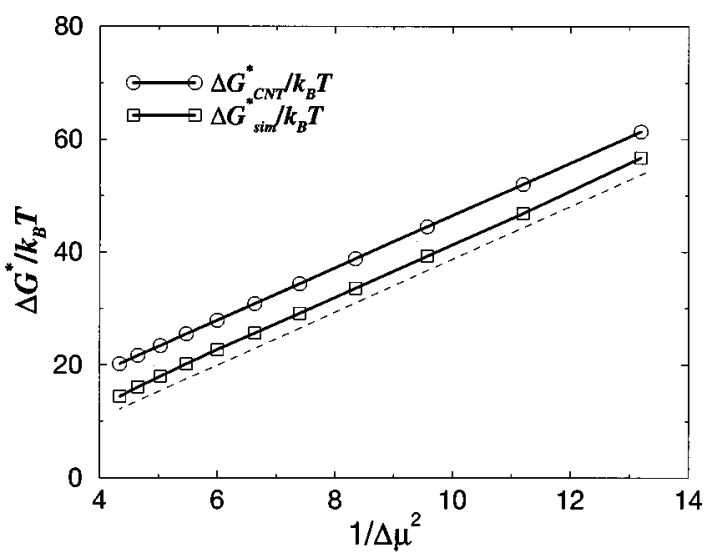

FIG. 10. The height of the barrier as predicted by classical nucleation theory and as obtained from the simulations, as a function of $1 / \Delta \mu^{2}$. The relations proposed by McGraw and Laaksonen (Refs. 17, 18) predict a constant offset between the actual barrier height and the height of the barrier as predicted by classical nucleation theory. The dashed curve is a guide to the eye; it has the same slope as the curve of the classical nucleation theory prediction.

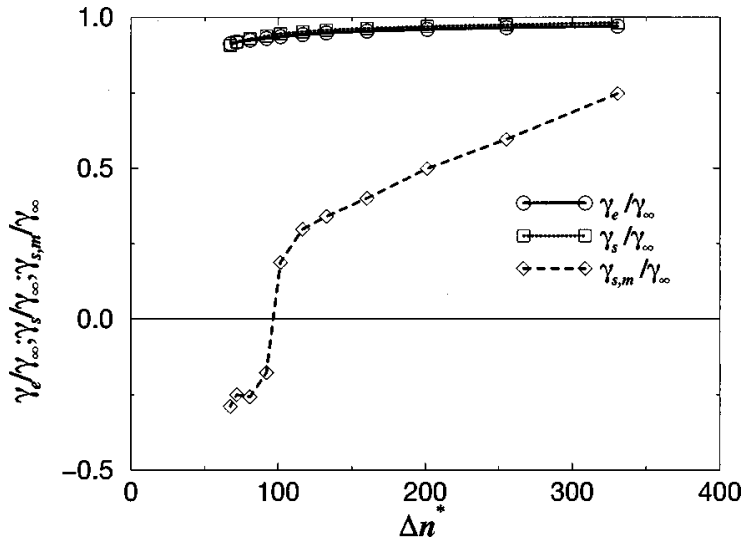

FIG. 11. The thermodynamically defined surface tension $\gamma_{s}$ and the mechanically defined surface tension $\gamma_{s, m}$ (acting at the respective surface of tensions), as well as the surface tension at the equimolar dividing surface, denoted by $\gamma_{e}$, as a function of the excess number of particles in the critical nuclei.

that the relations proposed in Refs. 17 and 18 are quite robust. If we have the right theoretical tools to predict the offset, a better agreement between experiment and (extended) CNT can be obtained. ${ }^{67}$

McGraw and Laaksonen ${ }^{18}$ showed that, within their nonuniform droplet model, the offset $D(T)$ is related to the rigidity coefficient $k_{s}$ :

$$
\Delta G_{\mathrm{CNT}}^{*}-\Delta G^{*}=D(T)=-4 \pi k_{s},
$$

where $k_{s} / R^{2}$ is the elastic curvature free-energy per unit area) From our simulations we estimate $k_{s}=-0.31 \epsilon$ $=-0.42 k_{\mathrm{B}} T$. This is smaller than the value that McGraw and Laaksonen obtained in their density functional calculations. ${ }^{18}$ However, the discrepancy could well be due to the fact that we used a truncated Lennard-Jones potential, whereas in the density functional calculations, the full Lennard-Jones potential was used.

\section{E. Tolman length}

We computed the thermodynamically defined surface tension and surface of tension using Eqs. (18) and (19). As explained in Sec. IV A, $\Delta p$ is the difference between the pressure in the vapor and the pressure in a hypothetical bulk liquid with a chemical potential equal to that of the vapor phase. We therefore performed a series of simulations of the bulk liquid to obtain the chemical potential of the liquid as a function of $P$. In Figs. 11 and 12, we show the thermodynamic surface tension and the location of the surface of tension, respectively. For small droplets, the surface tension increases rapidly with droplet size, and then smoothly approaches its planar limit. Such behavior has also been found in theoretical studies. ${ }^{35,61,62}$

For the sake of comparison, we have also calculated the mechanical surface tension and surface of tension using Eqs. (35) and (36). The results are shown in Figs. 11 and 12, respectively. The figures show that both the surface tension and the surface of tension become negative for clusters smaller than 100 particles. The reason for this can be understood from Fig. 7. As can be seen from this figure, small 


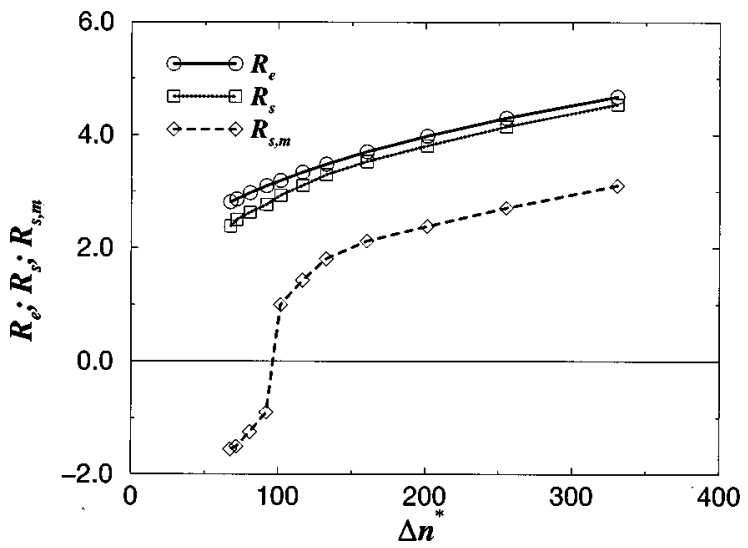

FIG. 12. The radii of the equimolar dividing surface, $R_{e}$, of the thermodynamically defined surface of tension, $R_{s}$ and of the mechanically defined surface of tension, $R_{s, m}$, as a function of the excess number of particles in the critical nuclei.

droplets have a progressively larger region where the pressure is lower than the vapor pressure. As discussed in Sec. VI B, this implies that the surface of small droplets is increasingly under compression. For clusters smaller than 100 particles, compression dominates and the integral in Eqs. (35) and (36) becomes negative. For a cluster size of about 100 , the integral in Eqs. (35) and (36) vanishes, and both $R_{s, m}$ and $\gamma_{s, m}$ cross zero.

Figure 12 also shows that the mechanically defined surface of tension and thermodynamically defined surface of tension are shifted with respect to one another. If we ignore the smallest droplets for which the radius of the mechanical surface tension becomes negative, the displacement is found to be constant over the range of droplet sizes studied, and equals approximately $1 \sigma$. It is thus clear that the two surfaces cannot be identified with each other. This was first pointed out by Blokhuis and Bedeaux, ${ }^{19}$ and later also found by Haye and Bruin ${ }^{36}$ in their computer simulation study of a planar interface. Haye and Bruin ${ }^{36}$ observed the displacement to be strongly depending on temperature, but for $T$ $=0.75$, which is quite close to the temperature of the present simulations, they also found a displacement close to $1 \sigma$, i.e., $0.92 \sigma$.

Figure 11 shows that not only the position of the surface of tension is different for the two definitions, but also the magnitude of the surface tension. In the planar limit the surface tensions should become equal, ${ }^{30,31}$ but for smaller droplets the difference becomes quite significant. In Fig. 11, we also show the surface tension at the equimolar dividing surface. McGraw and Laaksonen ${ }^{18}$ showed that within their nonuniform droplet model, the surface tension at the equimolar dividing surface can be obtained from the offset between the actual barrier height and the height of the barrier as predicted by CNT:

$$
\Delta G_{\mathrm{CNT}}^{*}-\Delta G=4 \pi R_{e}^{2}\left(\gamma_{\infty}-\gamma_{e}\right) .
$$

Here, $\gamma_{e}$ is the surface tension at the equimolar dividing surface and $R_{e}$ is the radius of the equimolar dividing surface, which is given by

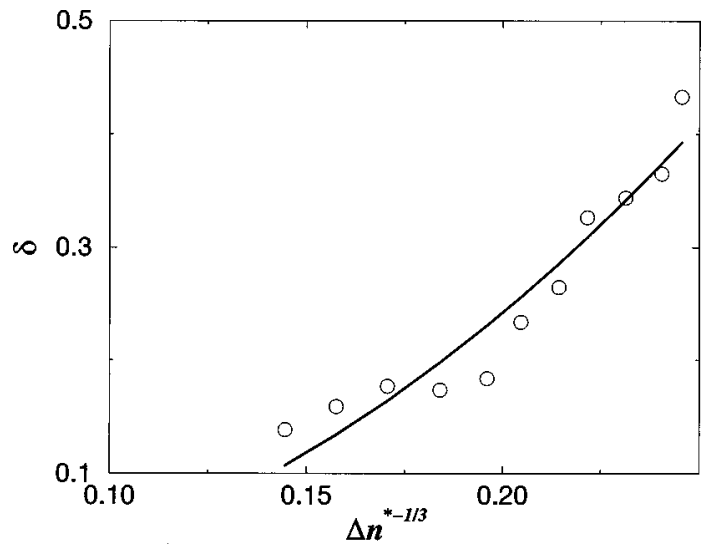

FIG. 13. The difference $\delta=R_{e}-R_{s}$ between the equimolar dividing surface and the surface of tension as a function of the excess number of particles, $\Delta n^{*-1 / 3}$, in the critical nuclei. The solid curve is a fit of the data to a function of the form $f\left(\Delta n^{*-1 / 3}\right)=c_{1} \Delta n^{*-1 / 3}+c_{2} \Delta n^{*-2 / 3}$. This corresponds to zero spontaneous curvature for the planar interface and to a Tolman length $\delta_{T}=\lim _{\Delta n^{*-1 / 3} \rightarrow 0} \delta=0.0$, as (implicitly) assumed in the nonuniform droplet model of McGraw and Laaksonen (Ref. 18). Clearly, our simulations do not rule out this possibility.

$$
R_{e}^{3}=\frac{3}{\rho(0)-\rho_{v}} \int\left[\rho(r)-\rho_{v}\right] r^{2} d r .
$$

The thermodynamically defined surface of tension is the surface for which the surface tension is at its minimum, ${ }^{30,31}$ hence, $\gamma_{s}$ should be smaller than $\gamma_{e}$. For the larger droplet sizes, the (Tolman) length $\delta \equiv R_{e}-R_{s}$ becomes small compared to the radius of the droplet. In that limit, we should expect the surface tensions to approach each other, as the surface tension varies quadratically with $R-R_{s} .{ }^{30,31}$ We find that $\gamma_{e}$ and $\gamma_{s}$ are equal to within the accuracy of our simulations.

In Fig. 13, we have plotted $\delta$ as a function of the size of the droplets. It is seen that $\delta$ is a strong function of the size of the droplet. In fact, our results are in fair agreement with the density functional calculations of Talanquer and Oxtoby. ${ }^{35}$ We can obtain the Tolman length by fitting $\delta$ to a polynomial and extrapolating the result to the planar limit. We find that $\delta_{T}$ is zero to within the accuracy of our simulations $\left(-0.2<\delta_{T}<0.8\right)$. Haye and Bruin $^{36}$ have computed the Tolman length for a range of temperatures by molecular dynamics simulations. Within the error bars, the Tolman length was found to be independent of temperature and equal to $\delta_{T}=0.16 \pm 0.04$, which is compatible with the earlier numerical calculations by Nijmeijer et al. ${ }^{26}$ who found that $\left|\delta_{T}\right|<0.7$ for $T=0.9$. Recently, Kalikmanov formulated a semiphenomenological cluster theory of the Tolman length based on the Fisher cluster model of condensation ${ }^{13}$ combined with a Tolman-like ansatz for the microscopic surface tension of a cluster. ${ }^{68}$ Kalikmanov performed calculations for a variety of nonpolar substances, ${ }^{68}$ which show that, not too close to the critical temperature $\left(\left|\left(T-T_{c}\right) / T_{c}\right|>0.1\right)$, the Tolman length is positive and about $0.2 \sigma$. Considering the small droplet sizes that we have studied, our results are in fair agreement with the previous results.

The Helfrich expression for the surface free-energy in powers of the inverse radius of curvature is ${ }^{19}$ 


$$
\gamma\left(R_{e}\right)=\gamma_{\infty}+2 k C_{0} \frac{1}{R_{e}}+(2 k+\bar{k})\left(\frac{1}{R_{e}}\right)^{2} .
$$

Here, $C_{0}$ is the spontaneous curvature, $k$ is the bending rigidity associated with the principal curvature, and $\bar{k}$ is the bending rigidity constant associated with Gaussian curvature. From the above expression it can be derived that the Tolman length is given by ${ }^{19}$

$$
\delta_{T}=-\frac{k C_{0}}{\gamma_{\infty}} .
$$

In the model of McGraw and Laaksonen, ${ }^{18}$ the surface freeenergy is given by

$$
\gamma\left(R_{e}\right)=\gamma_{\infty}+\frac{k_{s}}{R_{e}^{2}} .
$$

A comparison of the above equations shows that in the model of McGraw and Laaksonen, it is implicitly assumed that $k C_{0}$, and hence the Tolman length is zero. Our simulations suggest that, for the Lennard-Jones system, this is a reasonable assumption.

Comparing Eqs. (51) and (53) shows that $k_{s}=2 k+\bar{k}$. We stress that $k_{s}$ can be negative. The condition that must be fulfilled is that $\gamma\left(R_{e}\right)$ is positive. If $\gamma$ was not positive, it would be energetically favorable for a cluster to break up into smaller clusters. For vesicles, $\gamma_{\infty}$ is close to zero, and $k_{s}$ must be positive, which implies $-2 k<\bar{k}<0$. For the liquid Lennard-Jones droplets, however, $\gamma_{\infty}$ is positive, and $k_{s}$ can be negative. We note that the Helfrich expansion to second order, and the relation of McGraw and Laaksonen, break down if $\gamma\left(R_{e}\right)$ becomes negative. Then, higher-order terms in the expansion of the surface free-energy in the inverse radii have to be taken into account. However, in the present case this only occurs for droplets containing fewer than five to ten particles.

\section{CONCLUSIONS}

In our simulations, we have studied the structure and thermodynamics of the (pre) critical nuclei that play a role in the homogeneous nucleation of the liquid phase from the vapor. We found that the dependence of the size of the critical nucleus on the degree of supersaturation is in excellent agreement with the nucleation theorem. Furthermore, our simulations show that liquid-like clusters larger than 200 particles show bulk behavior in the core. That is, the pressure and the density in the core of the droplets are those of a bulk fluid with a chemical potential equal to that of the vapor phase. However, smaller droplets start to lose this bulk behavior. Nevertheless, the critical-nucleus sizes are still correctly predicted by classical nucleation theory. The simulation results for the height of the nucleation barrier differ by a constant amount from the prediction of classical nucleation theory, not only for large droplets, but even for quite small droplets. This constant offset can be accounted for by assuming that the surface tension depends quadratically on $1 / R^{17,18}$
For the range of droplet sizes studied here, we find a significant discrepancy between the thermodynamic and mechanical descriptions of the surface tension. Of course, one could argue that the thermodynamic description should fail for very small droplets. But even for larger droplets, which do show bulk liquid behavior in the core, we find that the respective surfaces of tension cannot be identified with each other, and that the surface tensions that follow from the different definitions are different. This implies that the height of the nucleation barrier cannot be obtained from Eq. (37). The failure of this equation is clearly illustrated by Fig. 5. The discrepancy between the free-energy barriers obtained by the thermodynamic and mechanical approaches is quite large, around $10-40 k_{\mathrm{B}} T$. In fact, as this figure shows, the mechanical route leads to an estimate for the height of the barrier that even becomes negative- this is due to the fact that the integral of the pressure profile in Eq. (37) becomes negative. Hence, at present, there seems to be no "cheap" numerical alternative to the direct (umbrella-sampling) approach to compute nucleation barriers.

\section{ACKNOWLEDGMENTS}

This work was supported in part by "Scheikundig Onderzoek Nederland" (SON) with financial aid from NWO ("Nederlandse Organisatie voor Wetenschappelijk Onderzoek'). The work of the FOM Institute is part of the research program of "Stichting Fundamenteel Onderzoek der Materie" (FOM) and is supported by NWO. We thank I. Pagonabarraga and E. Blokhuis for the stimulating discussions.

\section{APPENDIX A: CLUSTER SIZE DISTRIBUTION}

Consider a system in a volume $V$, at temperature $T$ and at constant chemical potential $\mu$ (i.e., a system in the grandcanonical ensemble). The partition function is given by

$$
\Xi(\mu, V, T) \equiv \sum_{N=0}^{\infty} \exp (\beta \mu N) Q(N, V, T),
$$

where $N$ is the number of particles, $\beta \equiv 1 / k_{\mathrm{B}} T$ is the reciprocal temperature, $k_{\mathrm{B}}$ is Boltzmann's constant, and $Q(N, V, T)$ is the canonical partition function:

$$
Q(N, V, T)=\frac{1}{\Lambda^{3 N} N !} \int d r^{N} \exp \left[-\beta U\left(r^{N}\right)\right] .
$$

Here, $U\left(r^{N}\right)$ is the potential energy of the configuration with the coordinates $r^{N}$, and $\Lambda \equiv h / \sqrt{2 \pi m k_{\mathrm{B}} T}$ is the thermal De Broglie wavelength.

Now we will assume that we have a criterion that enables us to define which particles make up a liquid cluster. The total number of particles in a liquid-like environment will be denoted by $N_{l}$, and the remaining particles in the vapor will be denoted by $N_{v}$; hence, $N=N_{l}+N_{v}$. Clearly, the potential energy $U$ depends on $r^{N_{v}}$ and $r^{N_{l}}$, i.e., $U$ $=U\left(r^{N_{l}} ; r^{N_{v}}\right)$, and we rewrite the grand-canonical partition function as 


$$
\begin{aligned}
& \Xi(\mu, V, T)=\sum_{N_{l}=0}^{\infty} \exp \left(\beta \mu N_{l}\right) \sum_{N_{v}=0}^{\infty} \exp \left(\beta \mu N_{v}\right) \\
& \times \frac{1}{\Lambda^{3 N_{v}} N_{v} !} \frac{1}{\Lambda^{3 N_{l}} N_{l} !} \\
& \times \int d r^{N_{v}} \int d r^{N_{l}} \mathscr{W}\left(r^{N_{l}} ; r^{N_{v}}\right) \\
& \times \exp \left[-\beta U\left(r^{N_{l}} ; r^{N_{v}}\right)\right],
\end{aligned}
$$

where we have used the fact that there are $N ! /\left(N_{l} ! N_{v} !\right)$ ways to select $N_{l}$ liquid-like and $N_{v}$ vapor-like particles from a total number of $N_{l}+N_{v}$ particles. In the above equation, we have introduced a weight function $\mathscr{W}$ which is defined such that it equals one when the number of particles that is liquid-like according to our definition equals $N_{l}$, and zero otherwise. It should be stressed that we do not assume that there is only a single liquid-like cluster in the system. Hence, we have to consider later the number of ways in which we can distribute $N_{l}$ particles over the total number of liquid clusters. In fact, $\mathscr{W}$ contains products of single-cluster weight functions. If we label the clusters by their size $n$ and by $j_{n}=1, \ldots, N_{n}$, where $N_{n}$ is the number of clusters of size $n$, it can be written as

$$
\mathscr{\mathscr { V }}_{N_{l}}=\sum \prod_{n} \prod_{j_{n}=1}^{N_{n}} w_{j_{n}}\left(r^{n}\right),
$$

where $\Sigma$ indicates that we consider all cluster distributions and $w_{j_{n}}$ equals one if its arguments satisfy the criterion for a single $j_{n}$-particle cluster, and zero otherwise. With these definitions, we can rewrite Eq. (A3) as

$$
\begin{aligned}
\Xi(\mu, V, T)= & \sum_{N_{1}=0}^{\infty} \sum_{N_{2}=0}^{\infty} \ldots \sum_{N_{n_{\max }}=0}^{\infty} \frac{1}{N_{1} ! N_{2} ! \cdots N_{n_{\max }} !} \prod_{n=1}^{n_{\max }}\left(\exp (\beta \mu n) n^{3} /\left[\Lambda^{3 n} n !\right]\right)^{N_{n}} \\
& \times \sum_{N_{v}=0}^{\infty} \exp \left(\beta \mu N_{v}\right) \frac{1}{\Lambda^{3 N_{v} N_{v} !}} \int d r^{N_{v}} \prod_{n}\left[\int d r^{\prime n-1}\right]^{N_{n}} \\
& \times \int_{n=1}^{n_{\max }} \prod_{j_{n}=1}^{N_{n}} d R_{j_{n}} w_{j_{n}}\left(R_{j_{n}}, r^{\prime n-1} ; r^{N_{v}}\right) \exp \left[-\beta U\left(R ; r^{N_{v}}\right)\right]
\end{aligned}
$$

Here, $R_{j_{n}}$ denotes the center-of-masses of the clusters and the primes indicate that the coordinates are taken with respect to the center-of-mass of the cluster. Note that we have not split the potential energy function yet. The product $\Pi_{n} \Pi_{j_{n}=1}^{N_{n}} w_{j_{n}}$ contains $\Pi_{n} N_{n}$ distinct cluster functions $w_{n}$. In this equation, we have somewhat arbitrarily introduced a maximum cluster size $n_{\max }$.

For any given configuration of clusters, we can define a potential of mean force $W\left(r^{N_{l}} ; \mu\right)$ as

$$
\begin{aligned}
& \exp \left[-\beta W\left(r^{\left.\left.N_{l} ; \mu\right)\right]}\right.\right. \\
& \equiv \sum_{N_{v}=0}^{\infty} \exp \left(\beta \mu N_{v}\right) \frac{1}{\Lambda^{3 N_{v}} N_{v} !} \\
& \quad \times \int d r^{N_{v}} \prod_{n=1}^{n_{\max }} \prod_{j_{n}=1}^{N_{n}} w_{j_{n}}\left(R_{j_{n}}, r^{\prime n-1} ; r^{N_{v}}\right) \\
& \quad \times \exp \left[-\beta U\left(r^{\left.\left.N_{l} ; r^{N_{v}}\right)\right] .}\right.\right.
\end{aligned}
$$

All possible configurations of the vapor particles will contribute to $W\left(r^{N_{l}} ; \mu\right)$. It is the average potential the particles in the liquid clusters feel due to all interactions with the "solvent" particles. With the above definition for the potential of mean force, the grand-canonical partition function can be rewritten as

$$
\begin{aligned}
\Xi(\mu, V, T)= & \sum_{N_{1}=0}^{\infty} \sum_{N_{2}=0}^{\infty} \cdots \sum_{N_{n_{\max }}=0}^{\infty} \frac{1}{N_{1} ! N_{2} ! \cdots N_{n_{\max }} !} \\
& \times \prod_{n=1}^{n_{\max }}\left(\exp (\beta \mu n) n^{3} /\left[\Lambda^{3 n} n !\right]\right)^{N_{n}} \\
& \times \prod_{n=1}^{n_{\max }}\left[\int d r^{\prime n-1}\right]^{N_{n}} \int \prod_{n=1}^{n_{\max }} \prod_{j_{n}=1}^{N_{n}} d R_{j_{n}} \\
& \times \exp \left[-\beta W\left(r^{\left.\left.N_{l} ; \mu\right)\right] .}\right.\right.
\end{aligned}
$$

The potential of mean force depends on the interactions between particles of the same cluster and on the interactions between particles of different clusters. In gas-liquid nucleation, the density of liquid clusters is usually so low that the interactions between them can be neglected. However, at this stage we will not yet ignore these, but assume that the intercluster interaction energy is pair-wise additive and only depends on the positions of the center-of-mass of the clusters. The interaction energy $W\left(r^{N_{l}} ; \mu\right)$ can then be written as

$$
\begin{aligned}
W\left(r^{N_{l}} ; \mu\right)= & W_{0}+\sum_{n} \sum_{j_{n}=1}^{N_{n}} W_{n}\left(r^{n, j_{n}} ; \mu\right) \\
& +\frac{1}{2} \sum_{n, n^{\prime}} \sum_{j_{n}, j_{n^{\prime}}} W_{n, n^{\prime}}\left(R_{n, j_{n}}, R_{n^{\prime}, j_{n}} ; \mu\right) .
\end{aligned}
$$


The constant $W_{0}$ is a measure for the grand potential in the absence of clusters. $W_{n}$ denotes the intracluster interaction energy of cluster $j_{n}$ of size $n$, and $W_{n, n^{\prime}}$ is a measure for the effective interaction between clusters $j_{n}$ and $j_{n^{\prime}}$. With this assumption for the potential of mean force, the grand partition function becomes

$$
\begin{aligned}
\Xi(\mu, V, T)= & \exp \left(-\beta W_{0}\right) \sum_{N_{1}=0}^{\infty} \sum_{N_{2}=0}^{\infty} \ldots \sum_{N_{n_{\max }}=0}^{\infty} \frac{1}{N_{1} ! N_{2} ! \ldots N_{n_{\max }} !} \prod_{n=1}^{n_{\max }}\left(\exp (\beta \mu n) n^{3} /\left[\Lambda^{3 n} n !\right]\right)^{N_{n}} \\
& \times \prod_{n=1}^{n_{\max }}\left[\int d r^{\prime n-1} \exp \left[-\beta W_{n}\left(r^{\prime n-1} ; \mu\right)\right]\right]^{N_{n}} \int \prod_{n=1}^{n_{\max }} \prod_{j_{n}=1}^{N_{n}} d R_{j_{n}} \exp \left[-\beta W_{n, n^{\prime}}\left(R_{n, j_{n}}, R_{n^{\prime}, j_{n^{\prime}}} ; \mu\right)\right] .
\end{aligned}
$$

As a final simplification, we ignore the interactions between the clusters. The partition function then becomes

$$
\begin{aligned}
\Xi(\mu, V, T)= & \exp \left(-\beta W_{0}\right) \sum_{N_{1}, N_{2}, \ldots,=0}^{\infty} \prod_{n}\left[\exp \left(\beta \mu n N_{n}\right)\right] \\
& \times \prod_{n} \frac{1}{N_{n} !}\left[\frac{V n^{3}}{\Lambda^{3 n} n !} \int d r^{\prime n-1}\right. \\
& \left.\times \exp \left[-\beta W_{n}\left(r^{\prime n-1} ; \mu\right)\right]\right]^{N_{n}} .
\end{aligned}
$$

We now define the partition function $Z_{n}$ of an $n$-mer, as

$$
Z_{n} \equiv \frac{V n^{3}}{\Lambda^{3 n} n !} \int d r^{\prime n-1} \exp \left[-\beta W_{n}\left(r^{\prime n-1} ; \mu\right)\right] .
$$

With the above definition of the partition function $Z_{n}$ of an $n$-mer, the grand partition function can be rewritten as

$$
\begin{aligned}
\Xi(\mu, V, T)= & \exp \left(-\beta W_{0}\right) \\
& \times \sum_{N_{1}, N_{2}, \ldots,=0}^{\infty} \prod_{n} \frac{\left[\exp (\beta \mu n) Z_{n}\right]^{N_{n}}}{N_{n} !} .
\end{aligned}
$$

We can interchange the order of the product and the summation to obtain for the partition function

$$
\begin{aligned}
\Xi(\mu, V, T) & =\exp \left(-\beta W_{0}\right) \prod_{n} \exp \left(\exp [\beta \mu n] Z_{n}\right) \\
& =\exp \left(-\beta W_{0}\right) \exp \left(\sum_{n} \exp [\beta \mu n] Z_{n}\right) .
\end{aligned}
$$

The average number of clusters of size $n$ is then simply given by

$$
\left\langle N_{n}\right\rangle=Z_{n} \exp [\beta \mu n] .
$$

\section{APPENDIX B: DETAILED BALANCE}

The detailed balance condition for the transition between state $i$ and $j$ is

$$
\rho_{i} P_{i \rightarrow j}^{\mathrm{gen}} P_{i \rightarrow j}^{\mathrm{acc}}=\rho_{j} P_{j \rightarrow i}^{\mathrm{gen}} P_{j \rightarrow i}^{\mathrm{acc}} .
$$

Here, $\rho_{i}$ is the Boltzmann weight of configuration $i, P_{i \rightarrow j}^{\mathrm{gen}}$ denotes the transition matrix which determines the probability to perform a trial move from state $i$ to state $j$, and $P_{i \rightarrow j}^{\text {acc }}$ is the probability with which this trial move is accepted.
In the standard Metropolis scheme, the transition matrix is symmetric and the acceptance criterion only depends on the Boltzmann factors of state $i$ and $j$. However, in the present scheme, liquid particles and vapor particles have different maximum displacements, which affects the transition matrix for the trial moves and needs to be taken into account in the acceptance criterion.

We rewrite Eq. (B1) as

$$
\frac{P_{i \rightarrow j}^{\mathrm{acc}}}{P_{j \rightarrow i}^{\mathrm{acc}}}=\frac{\rho_{j}}{\rho_{i}} \frac{P_{j \rightarrow i}^{\mathrm{gen}}}{P_{i \rightarrow j}^{\mathrm{gen}}} .
$$

The probability to generate a move from $i$ to $j$ is proportional to the inverse cube of the maximum displacement, denoted by drmax, and depends on the state of the particle, $s$. The acceptance criterion now becomes

$$
\frac{P_{i \rightarrow j}^{\mathrm{acc}}}{P_{j \rightarrow i}^{\mathrm{acc}}}=\frac{\rho_{j}}{\rho_{i}}\left(\frac{\operatorname{drmax}_{i}(s)}{\operatorname{drmax}_{j}(s)}\right)^{3} .
$$

There are many possible choices for $P_{i \rightarrow j}^{\text {acc }}$ that satisfy this condition (and the obvious condition that the probability cannot exceed 1). We have adopted the Metropolis rule

$$
P_{i \rightarrow j}^{\mathrm{acc}}=\operatorname{Min}\left[\frac{\rho_{j}}{\rho_{i}}\left(\frac{\operatorname{drmax}_{i}(s)}{\operatorname{drmax}_{j}(s)}\right)^{3}, 1\right] .
$$

However, there is one other condition that we have not mentioned yet. If a particle makes a transition from the vapor to the liquid and its displacement is larger than the maximum displacement in the liquid, then the move should be rejected. The reason is that when this move would have been accepted (and the particle would have become a liquid particle), the reverse move could never be made (because the maximum displacement for liquid particles is smaller than the maximum displacement for vapor particles).

\section{APPENDIX C: THE NUCLEATION THEOREM}

Consider a system in the grand-canonical ensemble. The probability to find $n$ particles in the system is given by

$$
P(n)=\exp (\beta \mu n) Q(n, V, T) / \Xi(\mu, V, T),
$$

where $\Xi$ is the grand-canonical partition function defined in Eq. (A1). The total grand potential $\Omega(=-P V)$ is given by

$$
\Omega=-k_{\mathrm{B}} T \ln \Xi .
$$

We can associate a Landau free-energy (strictly speaking, a Landau "grand potential") $\omega(n)$ with the probability distribution $P(n)$ 


$$
\begin{aligned}
\omega(n) & =-k_{\mathrm{B} T} \ln [\exp (\beta \mu n) Q(n, V, T)] \\
& =-\mu n+F(n, V, T),
\end{aligned}
$$

where $F(n, V, T)$ is the Helmholtz free-energy of a system of $n$ particles in volume $V$ at temperature $T$. The free-energy difference between two states with different numbers of particles, say $n_{1}$ and $n_{2}$, is

$$
\begin{aligned}
\Delta \omega & \equiv \omega\left(n_{2}\right)-\omega\left(n_{1}\right) \\
& =-\mu\left(n_{2}-n_{1}\right)+F\left(n_{2}, V, T\right)-F\left(n_{1}, V, T\right) .
\end{aligned}
$$

Let us now consider how $\Delta \omega$ varies with $\mu$. Note that the Helmholtz free-energy does not depend on $\mu$. Hence,

$$
\frac{\partial \Delta \omega}{\partial \mu}=-\left(n_{2}-n_{1}\right) \equiv-\Delta n .
$$

This result is general. Hence, it also holds for the case where $n_{1}$ corresponds to the (local) maximum of $P(n)$, i.e., the homogeneous metastable phase, while $n_{2}$ corresponds to the top of the (nucleation) barrier. In that case, it is immediately clear that $\Delta n$ corresponds to the excess number of particles in the critical nucleus. The extension to mixtures is straightforward.

In a sense, this result is so trivial it is easy to extend it to other ensembles. Consider first the $N, P, T$ ensemble. In that case, the fluctuating quantity is the volume, the Landau freeenergy has the form of a Gibbs free-energy, and we obtain

$$
\frac{\partial \Delta g}{\partial P}=\left(V_{2}-V_{1}\right) \equiv-\Delta V \text {. }
$$

Hence, the variation of the barrier height with pressure is given by the (usually negative) excess volume of the critical nucleus. However, although that quantity is well defined, it is intuitively not very appealing. Of course, if we use the Gibbs-Duhem relation to write $d P=\rho d \mu$, then Eq. (C6) reduces to Eq. (C5).

Finally, consider a variation in temperature, rather than pressure or chemical potential. Then, for both the grandcanonical and the isothermal-isobaric ensemble we obtain the same results:

$$
\frac{\partial \Delta g}{\partial T}=-\left(S_{2}-S_{1}\right) \equiv-\Delta S
$$

and

$$
\frac{\partial \beta \Delta g}{\partial \beta}=\left(E_{2}-E_{1}\right) \equiv \Delta E .
$$

It is worth pointing out that, in practice, the observable quantity is $\beta \Delta G$; hence the second relation is more useful.

Note that although $\Delta n$ in Eq. (C5) is a useful order parameter for small systems, it becomes less meaningful for larger systems. To be more specific, it becomes meaningless if the volume $V$ is so large that the spontaneous fluctuations in the number of particles become comparable to $\Delta n$. In general,

$$
\left\langle N^{2}\right\rangle-\langle N\rangle^{2}=N k_{\mathrm{B}} T \kappa,
$$

where $\kappa$ denotes the isothermal compressibility. For an ideal gas,

$$
\left\langle N^{2}\right\rangle-\langle N\rangle^{2}=N
$$

Hence, a problem arises when $n_{\text {nucleus }}^{*}$ is of order $\sqrt{N}$. When this happens, the change in free-energy associated with a small, homogeneous fluctuation in the density is smaller than the change in free-energy due to the formation of a liquidlike droplet, and $\Delta n$ is no longer a useful order parameter. Note that this situation can always arise when the volume is large enough and that it becomes even more serious close to the critical point or the spinodal. However, in practice, the problem is less serious because nucleation experiments do not probe the probability of arbitrary density fluctuations, but only those that result in the formation of a critical nucleus.

${ }^{1}$ M. Volmer and A. Weber, Z. Phys. Chem., Stoechiom. Verwandtschaftsl. 119, 227 (1926)

${ }^{2}$ R. Becker and W. Döring, Ann. Phys. (Leipzig) 24, 719 (1935).

${ }^{3}$ Y. Viisanen, R. Strey, and H. Reiss, J. Chem. Phys. 99, 4680 (1993).

${ }^{4}$ K. N. H. Looijmans, C. C. M. Luijten, and M. E. H. van Dongen, J. Chem. Phys. 103, 1714 (1995).

${ }^{5}$ D. Kashchiev, J. Chem. Phys. 76, 5098 (1984).

${ }^{6}$ D. W. Oxtoby and D. Kashchiev, J. Chem. Phys. 100, 7665 (1994).

${ }^{7}$ I. J. Ford, J. Chem. Phys. 105, 8324 (1996).

${ }^{8}$ R. Strey and Y. Viisanen, J. Chem. Phys. 99, 4693 (1993).

${ }^{9}$ Y. Viisanen, R. Strey, A. Laaksonen, and M. Kulmala, J. Chem. Phys. 100, 6062 (1994)

${ }^{10}$ R. Strey, Y. Viisanen, and P. E. Wagner, J. Chem. Phys. 103, 4333 (1995).

${ }^{11}$ J. Lothe and G. M. Pound, J. Chem. Phys. 36, 2080 (1962).

${ }^{12}$ H. Reiss, J. L. Katz, and E. R. Cohen, J. Stat. Phys. 2, 83 (1968).

${ }^{13}$ M. E. Fisher, Physics (Long Island City, NY) 3, 255 (1967).

${ }^{14}$ A. Dillmann and G. E. A. Meier, Chem. Phys. Lett. 89, 71 (1989).

${ }^{15}$ V. I. Kalikmanov and M. E. H. van Dongen, Europhys. Lett. 21, 645 (1993).

${ }^{16}$ D. W. Oxtoby and R. Evans, J. Chem. Phys. 89, 7521 (1988).

${ }^{17}$ R. McGraw and A. Laaksonen, Phys. Rev. Lett. 76, 2754 (1996).

${ }^{18}$ R. McGraw and A. Laaksonen, J. Chem. Phys. 106, 5284 (1997).

${ }^{19}$ E. M. Blokhuis and D. Bedeaux, J. Chem. Phys. 97, 3576 (1992).

${ }^{20}$ J. K. Lee, J. A. Barker, and F. F. Abraham, J. Chem. Phys. 58, 3166 (1973).

${ }^{21}$ D. J. McGinty, J. Chem. Phys. 58, 4733 (1973).

${ }^{22}$ M. Rao, B. J. Berne, and M. H. Kalos, J. Chem. Phys. 68, 1325 (1978).

${ }^{23}$ N. G. Garcia and J. M. S. Torroja, Phys. Rev. Lett. 47, 186 (1981).

${ }^{24}$ S. M. Thompson, K. E. Gubbins, J. P. R. B. Walton, R. A. R. Chantry, and J. S. Rowlinson, J. Chem. Phys. 81, 530 (1984).

${ }^{25}$ D. J. Lee, M. M. Telo da Gama, and K. E. Gubbins, J. Chem. Phys. 85, 490 (1986).

${ }^{26}$ M. J. P. Nijmeijer, C. Bruin, A. B. Woerkom, A. F. Bakker, and J. M. J. van Leeuwen, J. Chem. Phys. 96, 565 (1992).

${ }^{27}$ C. L. Weakliem and H. Reiss, J. Chem. Phys. 99, 5374 (1993).

${ }^{28}$ G. M. Torrie and J. P. Valleau, Chem. Phys. Lett. 28, 578 (1974).

${ }^{29}$ R. C. Tolman, J. Chem. Phys. 17, 333 (1949).

${ }^{30}$ S. Ono and S. Kondo, in Encyclopedia of Physics, edited by S. Flugge (Springer, Berlin, 1960), Vol. 10.

${ }^{31} \mathrm{~J}$. S. Rowlinson and B. Widom, Molecular Theory of Capillarity (Clarendon, Oxford, 1982).

${ }^{32}$ J. P. Hansen and L. Verlet, Phys. Rev. 184, 151 (1969).

${ }^{33}$ X. C. Zheng and D. W. Oxtoby, J. Chem. Phys. 94, 4472 (1991).

${ }^{34}$ V. Talanquer and D. W. Oxtoby, J. Chem. Phys. 100, 5190 (1994).

${ }^{35}$ V. Talanquer and D. W. Oxtoby, J. Phys. Chem. 99, 2865 (1995).

${ }^{36}$ M. J. Haye and C. Bruin, J. Chem. Phys. 100, 556 (1994).

${ }^{37}$ F. H. Stillinger, J. Chem. Phys. 38, 1486 (1963).

${ }^{38}$ H. Reiss, W. K. Kegel, and J. L. Katz, Phys. Rev. Lett. 78, 4506 (1997).

${ }^{39}$ H. Reiss, A. Tabazadeh, and J. Talbot, J. Chem. Phys. 92, 1266 (1990).

${ }^{40}$ H. M. Ellerby, C. L. Weakliem, and H. Reiss, J. Chem. Phys. 95, 9209 (1991).

${ }^{41}$ H. M. Ellerby and H. Reiss, J. Chem. Phys. 97, 5766 (1992).

${ }^{42}$ C. L. Weakliem and H. Reiss, J. Chem. Phys. 101, 2398 (1994).

${ }^{43}$ K. J. Oh, X. C. Zeng, and H. Reiss, J. Chem. Phys. 107, 1242 (1997).

${ }^{44}$ P. R. ten Wolde, M. J. Ruiz-Montero, and D. Frenkel, Faraday Discuss. 104, 93 (1996). 
${ }^{45}$ P. R. ten Wolde and D. Frenkel, Science 277, 1975 (1997).

${ }^{46}$ P. R. ten Wolde, M. J. Ruiz-Montero, and D. Frenkel, Phys. Rev. Lett. 75, 2714 (1995).

${ }^{47}$ P. R. ten Wolde, M. J. Ruiz-Montero, and D. Frenkel, J. Chem. Phys. 104, 9932 (1996).

${ }^{48}$ J. W. Gibbs, The Scientific Papers of J. Willard Gibbs (Dover, New York, 1961), Vol. 1.

${ }^{49}$ F. P. Buff, J. Chem. Phys. 23, 419 (1955).

${ }^{50}$ A. Laaksonen and R. McGraw, Europhys. Lett. 35, 367 (1996).

${ }^{51}$ P. Schofield and J. R. Henderson, Proc. R. Soc. London, Ser. A 379, 231 (1982).

${ }^{52}$ J. H. Irving and J. G. Kirkwood, J. Chem. Phys. 18, 817 (1950).

${ }^{53}$ A. Harasima, Adv. Chem. Phys. 1, 203 (1958).

${ }^{54}$ J. P. R. B. Walton, D. J. Tildesley, and J. S. Rowlinson, Mol. Phys. 48, 1357 (1983).

${ }^{55}$ I. Kusaka, Z.-G. Wang, and J. H. Seinfeld, J. Chem. Phys. 108, 3416 (1998).

${ }^{56}$ M. P. Allen and D. J. Tildesley, Computer Simulation of Liquids (Clarendon, Oxford, 1987).
${ }^{57}$ A. M. Ferrenberg and R. H. Swendson, Phys. Rev. Lett. 63, 1195 (1989).

${ }^{58}$ B. Smit, J. Chem. Phys. 96, 8639 (1992).

${ }^{59}$ G. A. Chapela, G. Saville, S. M. Thompson, and J. S. Rowlinson, J. Chem. Soc., Faraday Trans. 2 8, 1133 (1977).

${ }^{60}$ C. D. Holcomb, P. Clancy, and J. A. Zollweg, Mol. Phys. 78, 437 (1993).

${ }^{61}$ A. H. Falls, L. E. Scriven, and H. T. Davis, J. Chem. Phys. 75, 3986 (1981).

${ }^{62}$ G. H. Peters and J. Eggenbrecht, J. Phys. Chem. 95, 909 (1991).

${ }^{63}$ I. Hadjiagapiou, J. Phys.: Condens. Matter 6, 5303 (1994).

${ }^{64}$ R. M. Nyquist, V. Talanquer, and D. W. Oxtoby, J. Chem. Phys. 103, 1175 (1995).

${ }^{65}$ As in classical nucleation theory, the fluid is assumed to be incompressible; it could be argued that in order to make a consistent comparison, it is more natural to take the density of the fluid to be constant and equal to the coexisting density. However, as the fluid is indeed rather incompressible, the difference is negligible.

${ }^{66}$ A. Z. Panagiotopoulos, Mol. Phys. 61, 813 (1987).

${ }^{67}$ V. Talanquer, J. Chem. Phys. 106, 9957 (1997).

${ }^{68}$ V. I. Kalikmanov, Phys. Rev. E 55, 3068 (1997). 